\title{
A Compliant Hybrid Zero Dynamics Controller for Stable, Efficient and Fast Bipedal Walking on MABEL
}

\author{
Koushil Sreenath, Hae-Won Park, Ioannis Poulakakis, J. W. Grizzle
}

\begin{abstract}
The planar bipedal testbed MABEL contains springs in its drivetrain for the purpose of enhancing both energy efficiency and agility of dynamic locomotion. While the potential energetic benefits of springs are well documented in the literature, feedback control designs that effectively realize this potential are lacking. In this paper, we extend and apply the methods of virtual constraints and hybrid zero dynamics, originally developed for rigid robots with a single degree of underactuation, to MABEL, a bipedal walker with a novel compliant transmission and multiple degrees of underactuation. A time-invariant feedback controller is designed such that the closed-loop system respects the natural compliance of the open-loop system and realizes exponentially stable walking gaits. Five experiments are presented that highlight different aspects of MABEL and the feedback design method, ranging from basic elements such as stable walking and robustness under perturbations, to energy efficiency and a walking speed of $1.5 \mathbf{~ m} / \mathbf{s}(3.4 \mathbf{~ m p h})$. The experiments also compare two feedback implementations of the virtual constraints, one based on PD control as in (Westervelt et al., 2004), and a second that implements a full hybrid zero dynamics controller. On MABEL, the full hybrid zero dynamics controller yields a much more faithful realization of the desired virtual constraints and was instrumental in achieving more rapid walking.
\end{abstract}

Index Terms-Bipedal robots, Hybrid Systems, Zero Dynamics, Compliance.

\section{INTRODUCTION}

MABEL is a novel bipedal testbed at the University of Michigan. The robot is planar, with a torso, two legs with revolute knees, and four actuators. Two of its actuators are in series with large springs for the purpose of enhancing both energy efficiency and agility of locomotion. The actuators are housed in the torso and the legs are light, placing the center of mass of the robot significantly above the hips, as shown in Figure 1. A more detailed description of the robot has been presented in (Hurst et al., 2007; Hurst and Rizzi, 2008;

Koushil Sreenath and J. W. Grizzle are with the Control Systems Laboratory, Electrical Engineering and Computer Science Department, University of Michigan, Ann Arbor, MI 48109-2122, USA. \{koushils, grizzle\}@umich.edu

Hae-Won. Park is with the Mechanical Engineering Department, University of Michigan, Ann Arbor, MI 48109-2125, USA. parkhweumich.edu

Ioannis Poulakakis is with the Mechanical and Aerospace Engineering Department, Princeton University, Princeton, NJ 08544-5263, USA. poulakas@princeton. edu

This work is supported by NSF grants ECS-909300, ECS-0600869.

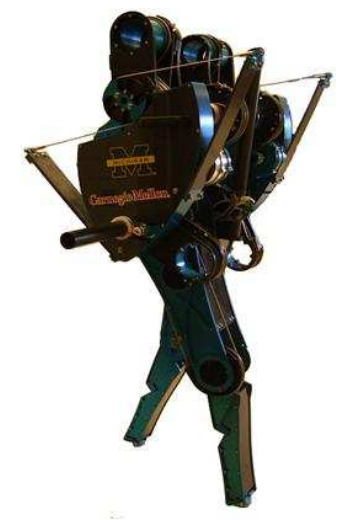

(a)

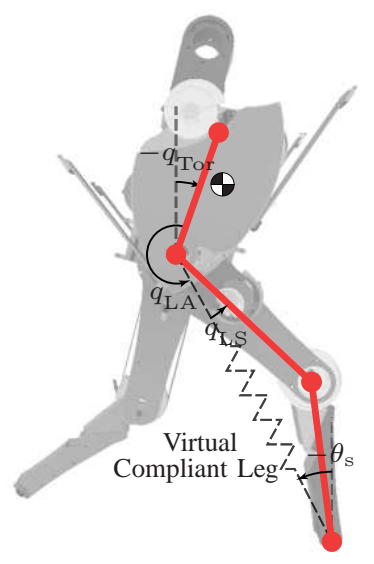

(b)
Fig. 1. (a) MABEL, an experimental testbed for bipedal locomotion. The robot is planar, with a boom providing stabilization in the frontal plane. The robot weighs $58 \mathrm{~kg}$ and is $1 \mathrm{~m}$ at the hip. The robot's drivetrain contains springs for enhanced power efficiency. (b) The virtual compliant leg created by the drivetrain through a set of differentials. The coordinate system used for the linkage is also indicated. Angles are positive in the counter clockwise direction.

Hurst, 2008), and the identification of its dynamic model is reported in (Park et al., 2010).

Bipedal robots that are simultaneously robustly stable, efficient, and fast are extremely rare. The desire to achieve these traits is driving the introduction of innovative mechanism designs and feedback control methods. MABEL was designed to be both a robust walker and a fast runner. It pushes the state of the art in bipedal mechanism design and provides an opportunity for effective control design methodology to maximize the robot's efficiency, speed and stability. This paper reports analytical and experimental results for walking on flat ground, a very important preliminary stage for running on flat ground and for walking on uneven ground. In particular, a Compliant Hybrid Zero Dynamics controller (HZD) is designed and the HZD controller is experimentally implemented to achieve stable, efficient, and fast walking.

\section{A. Background}

The stability of a biped can be enhanced by introducing large feet, in which case relatively simple controller designs can be used. Honda's ASIMO (Sakagami et al., 
2002), Sony's QRIO (Geppert, 2004), and the HRP series (Kaneko et al., 2002) have large feet and use zero moment point-based controllers (Vukobratović and Borovac, 2004) to achieve stable walking. The walking gaits are flat-footed and the achieved energy efficiency is low.

Enhanced agility has been demonstrated on hopperstyle robots (i.e., springy, prismatic leg) employing intuitive controllers, as demonstrated in (Hodgins and Raibert, 1990; Brown and Zeglin, 1998). These robots are highly underactuated, though for the most part, their control systems did not have to deal with stabilization of significant torso dynamics; indeed, if a torso was present, its center of mass was coincident with the hip joint (Poulakakis and Grizzle, 2009b).

The bipedal robot RABBIT was planar, had revolute knees, and a non-trivial torso (Chevallereau et al., 2003). It was deliberately designed to have point feet in order to inspire new analytical control approaches to stabilizing periodic motion in underactuated mechanical systems, and hence move beyond flat-footed walking gaits. Research on RABBIT gave rise to the methods of virtual constraints and hybrid zero dynamics (Grizzle et al., 2001; Westervelt et al., 2002, 2003; Morris and Grizzle, 2005; Westervelt et al., 2007), which provide a systematic method of designing asymptotically stable walking controllers. A related approach based on designing a linear feedback controller that stabilizes the time-varying transverse linearization of a hybrid system along a periodic orbit has been developed in (Manchester et al., 2009; Shiriaev et al., 2005, 2010; Song and Žefran, 2006). Other types of controllers to achieve stable walking are based on machine learning and neuronal control, as presented in (Russ Tedrake and Seung, 2005) and (Manoonpong et al., 2007; Sabourin et al., 2006), respectively.

The efficiency of bipedal robots is being enhanced by using minimal actuation, incorporating compliance, or a combination of the two. Motivated by passive dynamic walkers which exhibit stable gaits on small downward slopes, and where gravity compensates for energy losses at leg impacts, researchers have devised efficient means of walking on flat ground by injecting minimal amounts of energy at key points in the gait (Collins et al., 2005; Kuo, 2002). Another means of enhancing energy efficiency is by introducing compliant elements. The energetic benefits of springs in legged locomotion are well documented (Alexander, 1990). Springs can be used to store and release energy that otherwise would be lost as actuators do negative work, and springs can be used to isolate actuators from shocks arising from leg impacts with the ground. Although these benefits are more pronounced in running, compliance can also be used beneficially in walking (Geyer et al., 2006; Iida et al., 2007, 2008). Enhanced energy efficiency was shown using pneumatic artificial muscles in (Vanderborght et al., 2008a,b; Takum et al., 2008), using springs in series with motors in (Pratt and Pratt, 1998; Schaub et al., 2009), and using springs in parallel with motors in (Yang et al., 2008). A combination of both methods, minimalistic actuation and compliant elements, is employed in the Cornell Biped (Collins and Ruina, 2005), and the T.U. Delft bipeds TUlip and Flame (Hobbelen et al., 2008) in order to improve efficiency. The drawbacks of these highly efficient walkers are that they cannot lift their legs over obstacles, readily change speeds, or run.

The speed of a biped can be enhanced by careful mechanism and control design as suggested in (Koechling and Raibert, 1993), and demonstrated in robots such as RunBot (Manoonpong et al., 2007).

MABEL achieves stability, efficiency and speed through a combination of the novel design of its drivetrain and the analytical methods being developed to control it. The robot's drivetrain uses a set of differentials to create a virtual prismatic leg between the hip and the toe such that one actuator controls the angle of the virtual leg with respect to the torso, and another actuator controls its length. Moreover, the drivetrain also introduces a compliant element, a unilateral spring present in the transmission, that acts along the virtual leg in series with the actuator controlling the leg length. A controller that properly utilizes this natural compliant dynamics will lead to an efficient gait. Further, with the above mechanical design, it is possible to place all of the actuators in the torso, thereby making the legs relatively light and enabling rapid leg motion for fast gaits. More details on the design philosophy are available in (Grizzle et al., 2009; Hurst, 2008).

MIT's Spring Flamingo achieved stable, efficient and fast walking by employing series elastic actuators (SEAs) and a virtual model controller (Pratt and Pratt, 1998; Pratt, 2000; Pratt et al., 2001). The virtual model controller creates virtual components, such as springs, dashpots, etc., through carefully computed joint torques. This enables intuitive tuning of parameters of the controller, though no formal stability results exist. The spring in MABEL may seem similar to that in the SEA, however the resemblance is only superficial. The SEA is designed for force control and cannot store significant amounts of energy. MABEL's springs provide a revolute instantiation of a spring-loaded prismatic (pogo-stick) leg. They can easily absorb $150 \mathrm{~J}$ of energy (the equivalent of dropping the robot from a height of $25 \mathrm{~cm}$.) The spring in the SEA is several orders of magnitude smaller in size, and is used primarily for filtering and sensing of external forces, rather than energy storage.

The presence of compliance in MABEL's transmission has led to new control challenges that cannot be met with the initial theory developed for RABBIT. On the mathematical side, compliance increases the degree of underactuation, which in turn makes it more difficult to meet the invariance condition required for a hybrid zero dynamics to exist. This technical difficulty was overcome in (Morris and Grizzle, 2009) with a technique called a "deadbeat hybrid extension".

A second challenge arising from compliance is how 
to use it effectively. A first attempt in (Morris and Grizzle, 2006) at designing a controller for a biped with springs took advantage of the compliance along a steady state walking gait, but "fought it" during transients; the compliance was effectively canceled in the HZD (for details, see (Poulakakis and Grizzle, 2009b, p. 1790)). The problem of ensuring that the feedback action preserves the compliant nature of the system even during transients was studied in (Poulakakis and Grizzle, 2009b,a; Poulakakis, 2008) for the task of hopping in a monopod, where the HZD itself was designed to be compliant.

\section{B. Contributions}

The key results of the paper are summarized next. Firstly, a HZD-based controller is designed for walking such that the natural compliant dynamics is preserved in the closed-loop system (robot plus controller). This ensures that the designed walking gait uses the compliance to do negative work at impact, instead of it being done by the actuators, thereby improving the energy efficiency of walking. Stability analysis using the method of Poincaré is then carried out to check stability of the closed-loop system. Prior to experimentally testing the controller, simulations with various model perturbations are performed to establish robustness of the designed controller. The controller is then experimentally validated on MABEL.

Secondly, walking gaits are designed to optimize the energetic cost of mechanical transport (Collins et al., 2005; Collins and Ruina, 2005). This results in a gait that is more than twice as efficient on the testbed than a gait that we had designed by hand and reported in (Grizzle et al., 2009). The resulting cost of mechanical transport is approximately three times more efficient than RABBIT, and 12 times better than Honda's ASIMO, even though MABEL does not have feet. This puts MABEL's energy efficiency within a factor of two of T.U. Delft's Denise and a factor of three of the Cornell Biped, none of which can step over obstacles or run; it is also within a factor of two of the MIT Spring Flamingo which can easily step over obstacles but cannot run, and within a factor of three of humans, who can do all of the above.

Thirdly, in preparation for future running experiments, we turn our attention to fast walking, where each single support phase may be on the order of 300 to $350 \mathrm{~ms}$. Very precise control is needed for accurately implementing the virtual constraints of an HZD controller with these gait times. All experimental implementations of the virtual constraints reported to date have relied on local PD controllers (Westervelt et al., 2004). The zero dynamics controllers provide great tracking accuracy in theory, but are often criticized for being overly dependent on high model accuracy, and for being too complex to implement in real-time. Here we demonstrate, for the first time, an experimental implementation of a compliant HZD controller. The tracking accuracy attained is far better than the simple PD controllers used earlier.
Finally, we attack the problem of achieving fast walking. With a zero dynamics controller, we experimentally attain a top sustained walking speed of $1.5 \mathrm{~m} / \mathrm{s}(3.4$ mph.)

The remainder of the paper is organized as follows. Section II describes the general features of MABEL's morphology, and presents the mathematical hybrid model used for walking. Section III provides the systematic procedure based on virtual constraints that is used to design a suite of walking gaits. Section IV presents the design of two controllers to realize the gaits and studies the stability of the fixed points under the action of the proposed controllers. Section V describes the experiments performed to demonstrate the validity of the designed controllers. Section VI discusses various aspects of the robot and the feedback controllers revealed by the experiments. Finally, Section VII provides concluding remarks and briefly discusses future research plans.

\section{MABEL TESTBED}

This section presents details about the morphology of MABEL, and develops the appropriate mathematical models for the study of walking.

\section{A. Description of $M A B E L$}

MABEL is a planar bipedal robot comprised of five links assembled to form a torso and two legs with knees; see Figure 1. The robot weighs $58 \mathrm{~kg}$, is $1 \mathrm{~m}$ at the hip, and mounted on a boom of radius $2.25 \mathrm{~m}$. The legs are terminated in point feet. All actuators are located in the torso, so that the legs are kept as light as possible; this is to facilitate rapid leg swinging for running. Unlike most bipedal robots, the actuated degrees of freedom of each leg do not correspond to the knee and hip angles. Instead, for each leg, a collection of cable-differentials is used to connect two motors to the hip and knee joints in such a way that one motor controls the angle of the virtual leg consisting of the line connecting the hip to the toe, and the second motor is connected in series with a spring in order to control the length or shape of the virtual leg; see Figure 2. The reader is referred to (Park et al., 2010; Grizzle et al., 2009; Hurst, 2008) for more details on the transmission.

The springs in MABEL serve to isolate the reflected rotor inertia of the leg-shape motors from the impact forces at leg touchdown and to store energy in the compression phase of a running gait, when the support leg must decelerate the downward motion of the robot's center of mass; the energy stored in the spring can then be used to redirect the center of mass upwards for the subsequent flight phase, when both legs are off the ground. These properties (shock isolation and energy storage) enhance the energy efficiency of running and reduce the overall actuator power requirements. This is also true for walking as we will demonstrate experimentally. MABEL has a unilateral spring which 


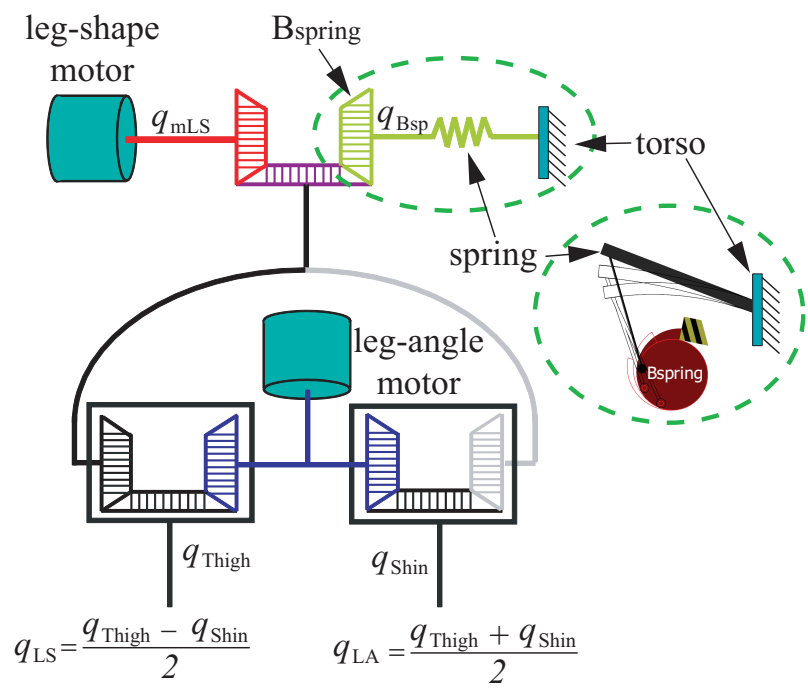

Fig. 2. MABEL's powertrain (same for each leg), all housed in the torso. Two motors and a spring are connected to the traditional hip and knee joints via three differentials. On the robot, the differentials are realized via cables and pulleys (Hurst, 2008) and not via gears. They are connected such that the actuated variables are leg angle and leg shape, see Figure 1, and so that the spring is in series with the leg shape motor. The base of the spring is grounded to the torso and the other end is connected to the $\mathrm{B}_{\text {spring }}$ differential via a cable, which makes the spring unilateral. When the spring reaches its rest length, the pulley hits a hard stop, formed by a very stiff damper. When this happens, the leg shape motor is, for all intents and purposes, rigidly connected to leg shape through a gear ratio.

compresses but does not extend beyond its rest length. This ensures that springs are present when they are useful for shock attenuation and energy storage, and absent when they would be a hindrance for lifting the legs from the ground.

\section{B. Mathematical Model}

A hybrid model appropriate for a walking gait, comprised of a continuous single support phase and an instantaneous double support phase, is developed next. The impact model at double support is based on (Hurmuzlu and Marghitu, 1994). The single support model is a pinned, planar, 5-link kinematic chain with revolute joints and rigid links. Because the compliance is unilateral, it will be more convenient to model it as an external force when computing the Lagrangian, instead of including it as part of the potential energy.

1) MABEL's Unconstrained Dynamics: The configuration space $Q_{\mathrm{e}}$ of the unconstrained dynamics of MABEL is a simply-connected subset of $\mathbb{S}^{7} \times \mathbb{R}^{2}$ : five DOF are associated with the links in the robot's body, two DOF are associated with the springs in series with the two leg-shape motors, and two DOF are associated with the horizontal and vertical position of the robot in the sagittal plane. A set of coordinates suitable for parametrization of the robot's linkage and transmission is $q_{\mathrm{e}}:=\left(q_{\mathrm{LA}_{\mathrm{st}}} ; q_{\mathrm{mLS}_{\mathrm{st}}} ; q_{\mathrm{Bsp}_{\mathrm{st}}} ; q_{\mathrm{LA} \mathrm{sw}_{\mathrm{w}}} ; q_{\mathrm{mLS}_{\mathrm{sw}}} ; q_{\mathrm{Bsp}_{\mathrm{sw}}}\right.$; $\left.q_{\text {Tor }} ; p_{\text {hip }}^{h} ; p_{\text {hip }}^{v}\right)$, the subscripts st and sw refer to the stance and swing legs respectively. As in Figure 1 and
Figure $2, q_{\text {Tor }}$ is the torso angle, and $q_{\mathrm{LA}_{\mathrm{st}}}, q_{\mathrm{mLS}}$, and $q_{\mathrm{Bsp}_{\mathrm{st}}}$ are the leg angle, leg-shape motor position, and $\mathrm{B}_{\text {spring }}$ position, respectively for the stance leg. The swing leg variables, $q_{\mathrm{LA}}, q_{\mathrm{mLS}}$ and $q_{\mathrm{Bsp}_{\mathrm{sw}}}$ are defined similarly. For each leg, $q_{L S}$ is determined from $q_{\mathrm{mLS}}$ and $q_{\text {Bsp }}$ by

$$
q_{L S}=0.0318 q_{\mathrm{mLS}}+0.193 q_{\mathrm{Bsp}} .
$$

This reflects the fact that the cable differentials place the spring in series with the motor, with the pulleys introducing a gear ratio. The coordinates $p_{\text {hip }}^{h}, p_{\text {hip }}^{v}$ are the horizontal and vertical positions of the hip in the sagittal plane. The hip position is chosen as an independent coordinate instead of the center of mass because it was observed that this choice significantly reduces the number of terms in the symbolic expressions for the dynamics.

The equations of motion are obtained using the method of Lagrange. The Lagrangian for the unconstrained system, $\mathcal{L}_{\mathrm{e}}: T Q_{\mathrm{e}} \rightarrow \mathbb{R}$ is defined by

$$
\mathcal{L}_{\mathrm{e}}=\mathcal{K}_{\mathrm{e}}-\mathcal{V}_{\mathrm{e}}
$$

where, $\mathcal{K}_{\mathrm{e}}: T Q_{\mathrm{e}} \rightarrow \mathbb{R}$ and $\mathcal{V}_{\mathrm{e}}: Q_{\mathrm{e}} \rightarrow \mathbb{R}$ are the total kinetic and potential energies of the mechanism, respectively. The total kinetic energy is obtained by summing the kinetic energy of the linkage, $\mathcal{K}_{\mathrm{e}}^{\text {link }}$, the kinetic energy of the stance and swing leg transmissions, $\mathcal{K}_{\mathrm{e}}^{\text {trans }} \mathrm{st}_{\mathrm{st}}, \mathcal{K}_{\mathrm{e}}^{\text {trans }} \mathrm{sw}_{\mathrm{sw}}$, and the kinetic energy of the boom, $\mathcal{K}_{\mathrm{e}}^{\text {boom }}$

$$
\begin{aligned}
\mathcal{K}_{\mathrm{e}}\left(q_{\mathrm{e}}, \dot{q}_{\mathrm{e}}\right)= & \mathcal{K}_{\mathrm{e}}^{\text {link }}\left(q_{\mathrm{e}}, \dot{q}_{\mathrm{e}}\right)+\mathcal{K}_{\mathrm{e}}^{\text {trans }_{\mathrm{st}}}\left(q_{\mathrm{e}}, \dot{q}_{\mathrm{e}}\right)+ \\
& \mathcal{K}_{\mathrm{e}}^{\text {trans }}{ }_{\mathrm{sw}}\left(q_{\mathrm{e}}, \dot{q}_{\mathrm{e}}\right)+\mathcal{K}_{\mathrm{e}}^{\text {boom }}\left(q_{\mathrm{e}}, \dot{q}_{\mathrm{e}}\right) .
\end{aligned}
$$

The linkage model is standard. Physically, the boom constrains the robot to move on the surface of a sphere, and a full 3D model would be required to accurately model the robot and boom system. However, we assume the motion to be planar and, as in (Westervelt, 2003, p. 94), only consider the effects due to mass and inertia of the boom. This will introduce some discrepancies between simulation and experimental results. The symbolic expressions for the transmission model are available online at (Grizzle, 2010b).

Similar notation is used for the potential energy,

$$
\begin{aligned}
\mathcal{V}_{\mathrm{e}}\left(q_{\mathrm{e}}\right)= & \mathcal{V}_{e}^{\text {link }}\left(q_{\mathrm{e}}\right)+\mathcal{V}_{\mathrm{e}}^{\text {trans }_{\mathrm{st}}}\left(q_{\mathrm{e}}\right)+ \\
& \mathcal{V}_{\mathrm{e}}^{\text {trans }} \mathrm{sw}_{\mathrm{sw}}\left(q_{\mathrm{e}}\right)+\mathcal{V}_{\mathrm{e}}^{\text {boom }}
\end{aligned}
$$

Due to its unilateral nature, the spring is not included in the potential energy of the transmission; only the mass of the motors and pulleys is included. The unilateral spring is considered as an external input to the system.

With the above considerations, the unconstrained robot dynamics can be determined through Lagrange's equations

$$
\frac{d}{d t} \frac{\partial \mathcal{L}_{\mathrm{e}}}{\partial \dot{q}_{\mathrm{e}}}-\frac{\partial \mathcal{L}_{\mathrm{e}}}{\partial q_{\mathrm{e}}}=\Gamma_{\mathrm{e}},
$$


where, $\Gamma_{\mathrm{e}}$ is the vector of generalized forces acting on the robot and can be written as,

$$
\begin{aligned}
\Gamma_{\mathrm{e}}= & B_{\mathrm{e}} u+E_{\text {ext }}\left(q_{\mathrm{e}}\right) F_{\mathrm{ext}}+ \\
& B_{\text {fric }} \tau_{\text {fric }}\left(q_{\mathrm{e}}, \dot{q}_{\mathrm{e}}\right)+B_{s p} \tau_{s p}\left(q_{\mathrm{e}}, \dot{q}_{\mathrm{e}}\right),
\end{aligned}
$$

where the matrices $B_{\mathrm{e}}, E_{\text {ext }}, B_{\text {fric }}$, and $B_{s p}$ are derived from the principle of virtual work and define how the actuator torques $u$, the external forces $F_{\text {ext }}$ at the leg, the joint friction forces $\tau_{\text {fric }}$, and the spring torques $\tau_{s p}$ enter the model, respectively.

Applying Lagrange's equations (5), with the kinetic and potential energies defined by (3) and (4), respectively, results in the second-order dynamical model

$$
D_{\mathrm{e}}\left(q_{\mathrm{e}}\right) \ddot{q}_{\mathrm{e}}+C_{\mathrm{e}}\left(q_{\mathrm{e}}, \dot{q}_{\mathrm{e}}\right) \dot{q}_{\mathrm{e}}+G_{\mathrm{e}}\left(q_{\mathrm{e}}\right)=\Gamma_{\mathrm{e}}
$$

for the unconstrained dynamics of MABEL. Here $D_{\mathrm{e}}$ is the inertia matrix, the matrix $C_{\mathrm{e}}$ contains Coriolis and centrifugal terms, and $G_{\mathrm{e}}$ is the gravity vector.

2) Dynamics of Stance: For modeling the stance phase, the stance toe is assumed to act as a passive pivot joint (no slip, no rebound and no actuation). Hence, the Cartesian position of the hip, $\left(p_{\text {hip }}^{h}, p_{\text {hip }}^{v}\right)$, is defined by the coordinates of the stance leg and torso. The springs in the transmission are appropriately chosen to support the entire weight of the robot, and hence are stiff. Consequently, it is assumed that the spring on the swing leg does not deflect, that is, $q_{\mathrm{Bsp}_{\mathrm{sw}}} \equiv 0$. It follows from (1) that $q_{\mathrm{mLS}_{\mathrm{sw}}}$ and $q_{\mathrm{LS}_{\mathrm{sw}}}$ are related by a gear ratio; $q_{\mathrm{mLS}_{\mathrm{sw}}}$ is taken as the independent variable. With these assumptions, the generalized configuration variables in stance are taken as $q_{\mathrm{s}}:=\left(q_{\mathrm{LA}_{\mathrm{st}}} ; q_{\mathrm{mLS}_{\mathrm{st}}} ; q_{\mathrm{Bsp}_{\mathrm{st}}} ; q_{\mathrm{LA}_{\mathrm{sw}}} ; q_{\mathrm{mLS}_{\mathrm{sw}}} ; q_{\mathrm{Tor}}\right)$.

The stance dynamics is obtained by applying the above holonomic constraints to the model of Section II-B1. The stance configuration space is therefore a co-dimension three submanifold of $Q_{\mathrm{e}}$, i.e., $Q_{\mathrm{s}}:=$ $\left\{q_{\mathrm{e}} \in Q_{\mathrm{e}} \mid q_{\mathrm{Bsp}_{\mathrm{sw}}} \equiv 0, p_{\text {toe }_{\mathrm{st}}}^{h} \equiv 0, p_{\text {toe }_{\mathrm{st}}}^{v} \equiv 0\right\}$. For later use, we denote by

$$
q_{\mathrm{e}}=\Upsilon_{\mathrm{s}}\left(q_{\mathrm{s}}\right)
$$

the value of $q_{\mathrm{e}}$ when $q_{\mathrm{s}} \in Q_{\mathrm{s}}$, and by

$$
q_{\mathrm{s}}=\Pi_{\mathrm{s}}\left(q_{\mathrm{e}}\right)
$$

the value of $q_{\mathrm{e}}$ projected onto $Q_{\mathrm{s}} \subset Q_{\mathrm{e}}$, such that, $\Pi_{\mathrm{s}} \circ$ $\Upsilon_{\mathrm{s}}=\mathrm{i} d$.

The resulting Lagrangian $\mathcal{L}_{\mathrm{s}}: T Q_{\mathrm{s}} \rightarrow \mathbb{R}$ can be expressed as

$$
\mathcal{L}_{\mathrm{s}}:=\left.\mathcal{L}_{\mathrm{e}}\left(q_{\mathrm{e}}, \dot{q}_{\mathrm{e}}\right)\right|_{\left\{q_{\mathrm{Bsp}_{\mathrm{sw}}} \equiv 0, p_{\text {toe }_{\mathrm{st}}}^{h} \equiv 0, p_{\text {toe }_{\mathrm{st}}}^{v} \equiv 0\right\}},
$$

and the dynamics of stance are obtained through Lagrange's equations, expressed in standard form as

$$
D_{\mathrm{s}}\left(q_{\mathrm{s}}\right) \ddot{q}_{\mathrm{s}}+C_{\mathrm{s}}\left(q_{\mathrm{s}}, \dot{q}_{\mathrm{s}}\right) \dot{q}_{\mathrm{s}}+G_{\mathrm{s}}\left(q_{\mathrm{s}}\right)=\Gamma_{\mathrm{s}},
$$

where, $\Gamma_{\mathrm{s}}:=B_{\mathrm{s}} u+B_{\text {fric }} \tau_{f r i c}\left(q_{\mathrm{s}}, \dot{q}_{\mathrm{s}}\right)+B_{s p} \tau_{s p}\left(q_{\mathrm{s}}, \dot{q}_{\mathrm{s}}\right)$ is the vector of generalized forces acting on the robot.
The state-space form of the stance dynamics, with the state vector $x_{\mathrm{s}}:=\left(q_{\mathrm{s}} ; \dot{q}_{\mathrm{s}}\right) \in T Q_{\mathrm{s}}$, can be expressed as,

$$
\begin{aligned}
\dot{x}_{\mathrm{s}} & :=\left[\begin{array}{l}
\dot{q}_{\mathrm{s}} \\
\ddot{q}_{\mathrm{s}}
\end{array}\right]=\left[\begin{array}{c}
\dot{q}_{\mathrm{s}} \\
-D_{\mathrm{s}}^{-1} H_{\mathrm{s}}
\end{array}\right]+\left[\begin{array}{c}
0 \\
D_{\mathrm{s}}^{-1} B_{\mathrm{s}}
\end{array}\right] u \\
& =: f_{\mathrm{s}}\left(x_{\mathrm{s}}\right)+g_{\mathrm{s}}\left(x_{\mathrm{s}}\right) u
\end{aligned}
$$

where, $f_{\mathrm{s}}, g_{\mathrm{s}}$ are the drift and input vector fields for the stance dynamics, and $H_{\mathrm{s}}:=C_{\mathrm{s}}\left(q_{\mathrm{s}}, \dot{q}_{\mathrm{s}}\right) \dot{q}_{\mathrm{s}}+G_{\mathrm{s}}\left(q_{\mathrm{s}}\right)-$ $B_{\text {fric }} \tau_{\text {fric }}\left(q_{\mathrm{s}}, \dot{q}_{\mathrm{s}}\right)-B_{s p} \tau_{s p}\left(q_{\mathrm{s}}, \dot{q}_{\mathrm{s}}\right)$.

3) Stance to Stance Transition Map: An impact occurs when the swing leg touches the ground, modeled here as an inelastic contact between two rigid bodies. In addition to modeling the impact of the leg with the ground and the associated discontinuity in the generalized velocities of the robot as in (Hurmuzlu and Marghitu, 1994), the transition map accounts for the assumption that the spring on the swing leg is at its rest length, and for the relabeling of robot's coordinates so that only one stance model is necessary. In particular, the transition map consists of three subphases executed in the following order: (a) standard rigid impact model (Hurmuzlu and Marghitu, 1994); (b) adjustment of spring rest length in the new swing leg; and (c) coordinate relabeling.

Before entering into the details, the spring is discussed. To meet our modeling assumption of Section II-B2, the post-transition spring position on the new swing leg has to be non-deflected. This requirement makes the pre and post-transition position coordinates not identical. Physically, the spring being non-deflected is a well-founded assumption because as soon as weight of the robot comes off the former stance leg, the spring rapidly relaxes and the pulley $q_{B s p}$ comes to rest on the hard stop. This causes a change in torque on the legshape motor, and either the motor shaft or the leg shape needs to reposition to maintain a balance of torques in the leg shape differentials. Because the leg shape has a high reflected inertia at the motor, it is the motor that repositions. Further, since $q_{\mathrm{LS}}$ is a linear combination of $q_{\mathrm{mLS}}$ and $q_{\mathrm{Bsp}}$ per (1), we can assume the spring and motor position change appropriately such that the linkage positions $q_{\mathrm{LS}}^{+}, q_{\mathrm{LS}}^{-}$are still identical. Thus, the pre and post-transition linkage coordinates still remain identical.

The robot physically transitions from one stance phase to the next when the swing toe contacts the ground. It is assumed that there is no rebound or slip at impact, and that the old stance leg lifts off from the ground without interaction. The external forces are represented by impulses, and since the actuators cannot generate impulses, they are ignored during impact. Mathematically, the transition then occurs when the solution of (12) intersects the co-dimension one switching manifold

$$
\mathcal{S}_{\mathrm{s} \rightarrow \mathrm{s}}:=\left\{x_{\mathrm{s}} \in T Q_{\mathrm{s}} \mid p_{\mathrm{toe}_{\mathrm{sw}}}^{v}=0\right\}
$$

The stance to stance transition map, $\Delta_{\mathrm{s} \rightarrow \mathrm{s}}: \mathcal{S}_{\mathrm{s} \rightarrow \mathrm{s}} \rightarrow$ 
$T Q_{\mathrm{s}}$, is defined as

$$
\Delta_{\mathrm{s} \rightarrow \mathrm{s}}:=\left[\begin{array}{c}
\Delta_{\mathrm{s} \rightarrow \mathrm{s}}^{q} \\
\Delta_{\mathrm{s} \rightarrow \mathrm{s}}^{\dot{q}}
\end{array}\right],
$$

where, the components $\Delta_{\mathrm{s} \rightarrow \mathrm{s}}^{q}$ and $\Delta_{\mathrm{s} \rightarrow \mathrm{s}}^{\dot{\mathrm{s}}}$ define the transition maps for the configuration variables and their velocities, respectively.

The transition map for the velocities is derived as follows. Let $I_{R}$ be the impulsive force on the foot due to the ground-foot impact and let $\tau_{R}$ be the impulsive torque on the spring due to $\mathrm{B}_{\text {spring }}$ hitting the hard stop ${ }^{1}$. Then the generalized external impulsive force acting on the system is obtained from the principle of virtual work as,

$$
F_{\mathrm{ext}}=\left(\frac{\partial p_{\mathrm{toe}_{\mathrm{sw}}}}{\partial q_{\mathrm{e}}}\right)^{\mathrm{T}} I_{R}+\left(\frac{\partial q_{\mathrm{Bsp}_{\mathrm{st}}}}{\partial q_{\mathrm{e}}}\right)^{\mathrm{T}} \tau_{R} .
$$

We have three constraints that need to be satisfied at impact. The first condition is for the new swing leg to have zero spring velocity. The second condition is for the new stance toe to have zero velocity. The third constraint is obtained by integrating the unconstrained dynamics, (7), over the duration of the instantaneous event. These conditions are

$$
\begin{gathered}
\dot{q}_{\mathrm{Bsp}_{\mathrm{st}}}^{+}=0 \Longrightarrow \frac{\partial q_{\mathrm{Bsp}_{\mathrm{st}}}}{\partial q_{\mathrm{e}}} \dot{q}_{\mathrm{e}}^{+}=0, \\
\dot{p}_{\mathrm{to}}^{+}=0 \Longrightarrow \frac{\partial p_{\mathrm{sw}}}{\partial q_{\mathrm{e}}} \dot{q}_{\mathrm{e}}^{+}=0, \\
D_{\mathrm{e}}\left(q_{\mathrm{e}}^{+}\right) \dot{q}_{\mathrm{e}}^{+}-D_{\mathrm{e}}\left(q_{\mathrm{e}}^{-}\right) \dot{q}_{\mathrm{e}}^{-}=F_{\mathrm{ext}} .
\end{gathered}
$$

By assembling the constraints (16)-(18), and solving for the post-impact velocity, we can define a map, $\Gamma$, such that, $\dot{q}_{\mathrm{e}}^{+}=\Gamma\left(\dot{q}_{\mathrm{e}}^{-}\right)$. Thus, the transition map for the velocities is

$$
\Delta_{\mathrm{s} \rightarrow \mathrm{s}}^{\dot{q}}:=\frac{\partial \Pi_{\mathrm{s}}}{\partial q_{\mathrm{e}}} \circ R \circ \Gamma \circ \frac{\partial \Upsilon_{\mathrm{s}}}{\partial q_{\mathrm{s}}},
$$

where $\Upsilon_{\mathrm{s}}, \Pi_{\mathrm{s}}$ are as defined in (8), (9), and $R$ is a linear operator representing coordinate relabeling as found in (Westervelt et al., 2007, p. 57).

Next, as per earlier discussions regarding the adjustment of spring rest length for the new swing leg, the transition map for the coordinates can be expressed as

$$
\Delta_{\mathrm{s} \rightarrow \mathrm{s}}^{q}:=\Pi_{\mathrm{s}} \circ R \circ T_{\mathrm{mLS}_{\mathrm{st}}} \circ \Upsilon_{\mathrm{s}},
$$

where $T_{\mathrm{mLS}}$ st resets the spring to its rest position by modifying the stance leg-shape motor position such that the stance leg-shape position itself is unchanged.

\footnotetext{
${ }^{1}$ We have checked that first doing the standard impact for the swing leg, and then doing a second impact for $q_{B s p}$ hitting the hard stop, with the constraint that the new stance leg end velocity remains zero, gives the same result as the model presented here.
}

4) Hybrid Model of Walking: The hybrid model of walking is based on the dynamics developed in Section II-B2 and transition map derived in Section II-B3. The continuous dynamics with discrete state transitions is represented as,

$$
\Sigma_{\mathrm{s}}: \begin{cases}\dot{x}_{\mathrm{s}}=f_{\mathrm{s}}\left(x_{\mathrm{s}}\right)+g_{\mathrm{s}}\left(x_{\mathrm{s}}\right) u & x_{\mathrm{s}}^{-} \notin S_{\mathrm{s} \rightarrow \mathrm{s}} \\ x_{\mathrm{s}}^{+}=\Delta_{\mathrm{s} \rightarrow \mathrm{s}}\left(x_{\mathrm{s}}^{-}\right) & x_{\mathrm{s}}^{-} \in S_{\mathrm{s} \rightarrow \mathrm{s}} .\end{cases}
$$

\section{Gait Design using Zero Dynamics}

This section presents a feedback controller for achieving asymptotically stable, periodic walking gaits on MABEL. In addition to orbital stability, a key objective is to take advantage of the spring in the robot's drivetrain that is placed in series with the leg-shape motor and $q_{\mathrm{LS}}$. Inspired by analysis in (Poulakakis and Grizzle, 2009b, p. 1784) and (Poulakakis, 2008, Chap. 6) for monopedal hoppers with compliance, this will be accomplished by controlling variables on the motor end of the spring and letting the joint end of the spring, which sees the large ground reaction forces, remain passive. In this way, the robot in closed-loop with the controller will respond to impulsive forces at impact in a manner similar to a pogo stick. In particular, the closed-loop system will use the compliance to do negative work at impact (i.e., decelerating the center of mass and redirecting it upward ${ }^{2}$ ), instead of it being done by the actuators, thereby improving the energy efficiency of walking.

It will be shown that the method of virtual constraints and hybrid zero dynamics is flexible enough to accomplish the control objectives outlined above. The method of Poincaré is used to verify stability of the closed-loop system. Prior to experimentally testing the controller, simulations with various model perturbations are performed to establish robustness of the designed controller. The controller is then experimentally validated on MABEL.

The rest of the section is as follows: Section III-A presents the virtual constraint design for walking, Section III-B presents the compliant zero dynamics for MABEL, Section III-C mathematically formalizes the event transitions between the subphases of the virtual constraints, Section III-D presents two cost functions for optimization and, finally, Section III-E presents the fixed points obtained by optimization.

\section{A. Virtual Constraint Design for Stance}

Recall that virtual constraints are holonomic constraints on the robot's configuration variables that are asymptotically imposed through feedback control. They are used to synchronize the evolution of the robot's links throughout a stride in order to synthesize a gait (Westervelt et al., 2007). One virtual constraint is designed per independent actuator.

\footnotetext{
${ }^{2}$ The double support phase of human walking is spring-like in the vertical direction and redirects the center of mass (Rebula et al., 2009; Geyer et al., 2006). The COM redirection is obtained here without a double support phase.
} 
The virtual constraints are parametrized by $\theta_{\mathrm{s}}$, a strictly monotonic function of the joint configuration variables, and can be expressed in the form

$$
y=h_{\mathrm{s}}\left(q_{\mathrm{s}}\right)=H_{0}^{\mathrm{s}} q_{\mathrm{s}}-h_{d}^{\mathrm{s}}\left(\theta_{\mathrm{s}}\right) .
$$

If a feedback can be found such that $y$ is driven asymptotically to zero, then $H_{0}^{\mathrm{s}} q_{\mathrm{s}} \rightarrow h_{d}^{\mathrm{s}}\left(\theta_{\mathrm{s}}\right)$ and thus the controlled variables $H_{0}^{\mathrm{s}} q_{\mathrm{s}}$ evolve according to the constraint $H_{0}^{\mathrm{s}} q_{\mathrm{s}}=h_{d}^{\mathrm{s}}\left(\theta_{\mathrm{s}}\right)$. Here, the controlled variables are selected to be the rotor angle of the stance leg-shape motor, $q_{\mathrm{mLS}}$, the swing leg variables, $q_{\mathrm{LA}}, q_{\mathrm{mLS}_{\mathrm{sw}}}$, and the absolute torso angle $q_{\text {Tor }}$. From hereon, the rotor angle of the stance leg-shape motor is simply referred to as stance motor leg shape.

1) Deciding what to control: The torso is selected as a controlled variable instead of the stance leg angle, because, for MABEL, the torso represents over $65 \%$ of the mass of the robot, and hence the position of the torso heavily influences the gait. The stance motor leg shape, $q_{\mathrm{mLS}}$ st is chosen instead of the stance leg shape, $q_{\mathrm{LS}}$, so that the joint side of the spring remains passive, as discussed above. Mathematically, with this choice, the spring variable will become a part of the zero dynamics, thereby rendering the zero dynamics compliant. From (1), if $q_{\mathrm{mLS}}$ is held constant, then $q_{\mathrm{LS}}$ responds to the spring torque through $q_{\mathrm{Bsp}_{\mathrm{st}}}$. On the other hand, if $q_{\mathrm{LS}}$ were selected as a controlled variable, then the actuator is forced to cancel the spring dynamics.

The swing leg virtual constraints are similar to the controlled variables on RABBIT, a robot without compliance. This is because under the assumption that the swing spring is at its rest position throughout stance, $q_{\mathrm{Bsp}_{\mathrm{sw}}} \equiv 0$, which from (1) shows that the motor leg shape, $q_{\mathrm{mLS}_{\mathrm{sw}}}$, is related to the leg shape, $q_{\mathrm{LS}}$, through a gear ratio.

In summary, the controlled variables are

$$
H_{0}^{\mathrm{s}} q_{\mathrm{s}}=\left[\begin{array}{c}
q_{\mathrm{mLS}_{\mathrm{st}}} \\
q_{\mathrm{LA}_{\mathrm{sw}}} \\
q_{\mathrm{mLS}_{\mathrm{sw}}} \\
q_{\mathrm{Tor}}
\end{array}\right],
$$

The desired evolution of each of the controlled variables are denoted by $h_{\mathrm{mLS}_{\mathrm{st}}}^{d}, h_{\mathrm{LA}_{\mathrm{sw}}}^{d}, h_{\mathrm{mLS}}^{d}$, and $h_{\text {Tor }}^{d}$ respectively, and assembled as

$$
h_{d}^{\mathrm{s}}\left(\theta_{\mathrm{s}}\right)=\left[\begin{array}{c}
h_{\mathrm{mLS}_{\mathrm{st}}}^{d}\left(\theta_{\mathrm{s}}\right) \\
h_{\mathrm{LA}_{\mathrm{sw}}}^{d}\left(\theta_{\mathrm{s}}\right) \\
h_{\mathrm{mLS}_{\mathrm{sw}}}^{d}\left(\theta_{\mathrm{s}}\right) \\
h_{\mathrm{Tor}}^{d}\left(\theta_{\mathrm{s}}\right)
\end{array}\right] .
$$

For MABEL, we choose $\theta_{\mathrm{s}}$ to be the absolute angle formed by the virtual compliant leg relative to the ground, i.e.,

$$
\theta_{\mathrm{s}}\left(q_{\mathrm{s}}\right)=\pi-q_{\mathrm{LA}_{\mathrm{st}}}-q_{\text {Tor }} .
$$

2) Specification of the constraints: Virtual constraints for the stance phase of MABEL are inspired by the constraints designed for Thumper in (Poulakakis and Grizzle, 2009a; Poulakakis, 2008). The stance phase is broken up into subphases: the motor-compression phase $(m c)$, the stance-compression phase $(s c)$, the stanceinjection phase (si), and the stance-decompression phase $(s d)$. The details of these subphases are given later in the section. Figure 3 illustrates the evolution of each of these constraints on $q_{\mathrm{mLS}_{\mathrm{st}}}, q_{\mathrm{LA}_{\mathrm{sw}}}, q_{\mathrm{mLS}_{\mathrm{sw}}}$, and $q_{\mathrm{Tor}}$.

The reason behind breaking up the stance phases into four subphases is to facilitate the design of virtual constraints that effectively make use of the compliance. A key idea is to hold the stance motor leg shape at a constant value shortly after impact in order to allow the spring (which is in series with this actuator) to absorb the impact shock entirely. Note that if the motor position is held constant, then its velocity is zero and the motor performs no mechanical work. The spring then does the negative work of decelerating the center of mass and redirecting it upwards; in other words, the spring stores the impact energy and returns it later to the gait instead of the actuator doing negative work and dissipating it as heat. This effectively preserves the natural compliant dynamics of the system and prevents the actuator from fighting the spring.

Another key subphase involves the torso. Because it is heavy, we have observed that making the pre-impact torso velocity close to zero at the end of the gait helps in avoiding excessive forward pitching of the torso just after swing leg impact. This is achieved by designing the torso virtual constraint such that, before impact, the torso position is constant and its velocity is zero; see phase $s$ d in Figure 3.

Remark 1: The choice of the variables to be controlled in the virtual constraints makes the zero dynamics compliant. The choice of the evolution of the virtual constraints facilitates efficient use of the compliance.

In our design of the virtual constraints for MABEL, we use the framework for virtual constraints with subphases developed in Appendix A, with the index set for the subphases given by

$$
\mathcal{P}:=\{m c, s c, s i, s d\},
$$

and with the index set for the virtual constraints given by

$$
\mathcal{V}:=\left\{\mathrm{mLS}_{\mathrm{st}}, \mathrm{LA}_{\mathrm{sw}}, \mathrm{mLS}_{\mathrm{sw}}, \text { Tor }\right\}
$$

Further, we choose $M=5$ in (52), and impose $\mathcal{C}^{1}$ continuity between successive subphases. This ensures continuity of position and velocity at the boundary of two phases of a virtual constraint. However, acceleration, and consequently, the actuator torques, are allowed to be discontinuous at phase boundaries.

3) Stance motor leg-shape virtual constraint: The desired evolution of the stance motor leg-shape position, $h_{\mathrm{mLS}}^{d}$, is as follows. During the motor-compression phase, the velocity of the motor leg-shape immediately after impact, $\dot{q}_{\mathrm{mLS}}^{\mathrm{s}+}$, is usually nonzero and is smoothly brought to zero by the end of the motor-compression phase, i.e., $\dot{q}_{\mathrm{mLS}}^{m c-}=0$. 


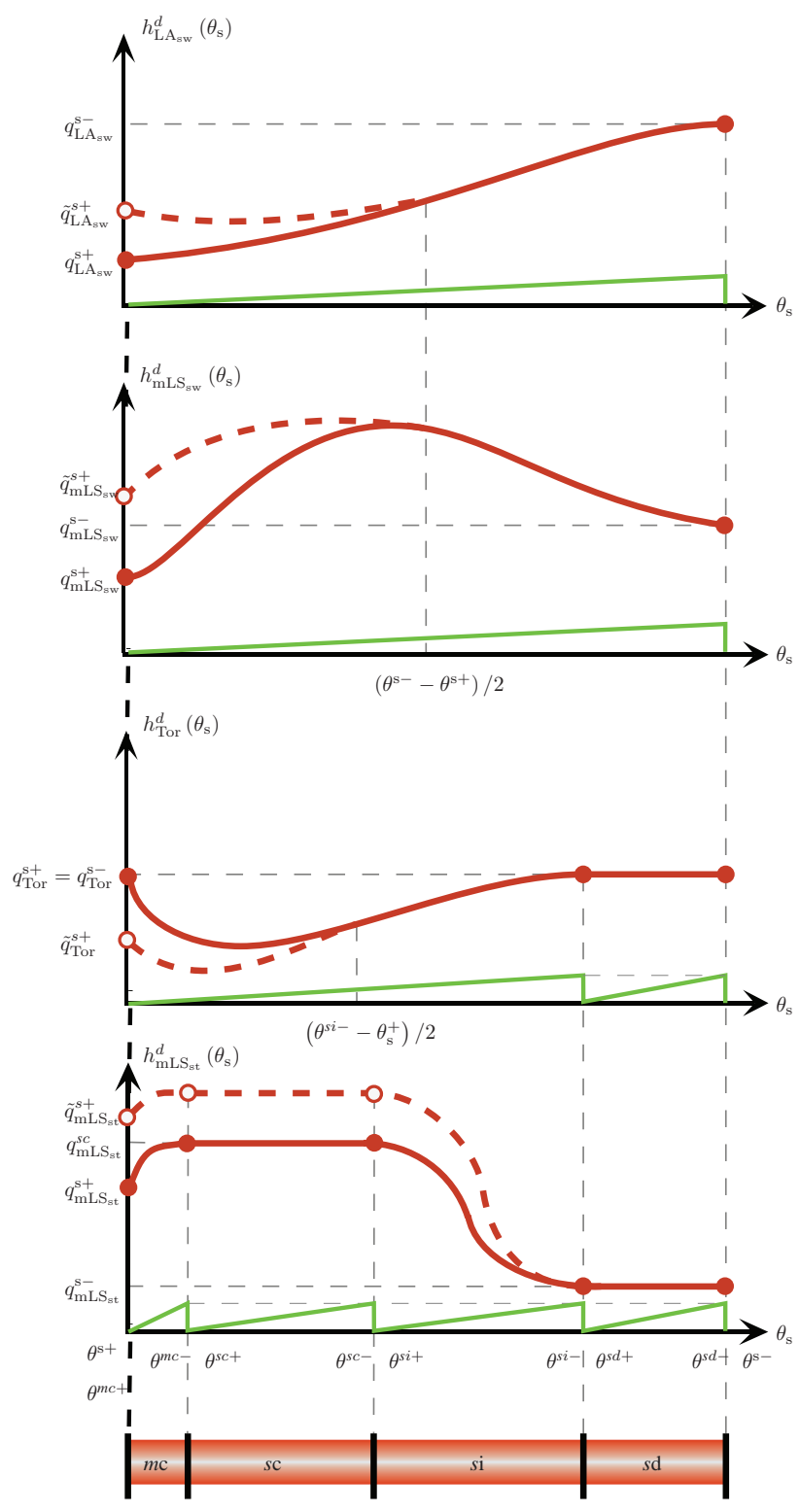

Fig. 3. The general shape of the stance phase virtual constraints. The thick solid lines illustrate the evolution of each of the virtual constraints as a function of $\theta_{\mathrm{s}}$. Each virtual constraint is broken into subphases $(m \mathrm{c}, s \mathrm{c}, s \mathrm{i}, s \mathrm{~d})$ and each subphase is locally expressed by a $5^{t h}$ order Bézier polynomial. The thin lines show the evolution of corresponding local $s$ that parametrizes the local Bézier curve and goes from 0 to 1 . The subphases can be combined as, for instance, in the torso virtual constraint, which lumps the first three subphases together and normalizes $s$ appropriately. The swing leg virtual constraints combine all four phases. The thick dashed lines are correction polynomials introduced to create hybrid invariance of the zero dynamics and are discussed in Section IV

Throughout the stance-compression phase, the legshape motor position is kept at a constant angle $q_{\mathrm{mLS}}^{s c}$. With the motor position locked, the bending of the stance knee compresses the spring. The phase lasts until $q_{\mathrm{Bsp}_{\mathrm{st}}}=q_{\mathrm{Bsp}_{\mathrm{st}}}^{s c+}$ with $\dot{q}_{\mathrm{Bsp}}<0$, the point at which the spring decompresses to a value near the nominal spring compression at mid-stance, a typical value being five degrees. This ensures that the impact kinetic energy is first stored in the spring and then returned to the gait without the actuator performing unnecessary negative work on the leg shape, $q_{\mathrm{LS}}$ st

The stance-injection phase starts with the spring just decompressed to $q_{\mathrm{Bsp}_{\mathrm{st}}}^{s c+}$. The actuator then rapidly repositions the motor shaft to a new desired position, $q_{\mathrm{mLS}}^{\mathrm{s}-}$. Under nominal conditions, this straightens the leg during mid-stance for ground clearance of the swing leg. Under large perturbations, this motion will cause the actuator to inject (or remove) energy through compression (or decompression) of the spring by rapidly repositioning the motor end of the compliance.

Following the stance-injection phase, the motor shaft is maintained at the position $q_{\mathrm{mLS}}^{\mathrm{s}-}$ throughout the stance-decompression phase, waiting for the spring to decompress again in preparation for leg touchdown.

The virtual constraints for the stance motor leg shape are depicted in Figure 3. The thick solid line is the virtual constraint, and the thin line is the local $s$ that parametrizes the local Bézier polynomial. The figure also shows the virtual constraints for the other controlled variables. Appendix A provides further details regarding choosing the the Bézier polynomial coefficients for each subphase.

4) Torso virtual constraint: The desired evolution of the torso angle, $h_{\text {Tor }}^{d}$, does not need to be as finely specified; it's evolution will be primarily left to optimization, which will be discussed in Section III-D. The motor-compression, stance-compression, and stance-injection phases, are combined into a single phase. This phase serves as a transient phase that drives the torso in a smooth manner from the initial configuration, $\left(q_{\text {Tor }}^{\mathrm{s}+}, \dot{q}_{\text {Tor }}^{\mathrm{s}+}\right)$, to the final configuration, $\left(q_{\text {Tor }}^{\mathrm{s}-}, \dot{q}_{\text {Tor }}^{\text {s- }}=0\right)$, in preparation for impact.

During the stance-decompression phase, the torso is held constant in preparation for impact. Simulations with the model and experiments with a simpler PD controller detailed in (Grizzle et al., 2009) showed that achieving a nearly zero pre-impact velocity tends to prevent the heavy torso from excessively pitching after impact.

5) Swing leg virtual constraints: The desired evolution of the swing leg angle, $h_{\mathrm{LA}_{\mathrm{sw}}}^{d}$, and motor leg shape position, $h_{\mathrm{mLS}}^{d}$ are the simplest of all of the constraints as no subphases are used. A single virtual constraint on swing leg angle is designed to bring the leg forward, preparing it for impact with a desired step length. The constraint on swing motor leg shape is responsible for lifting the swing leg from the ground, avoiding foot scuffing during the gait, and extending the leg before impact. These two constraints are similar to RABBIT (Westervelt et al., 2004).

6) Discussion: The use of subphases in the evolution of the stance motor leg shape and torso introduces additional independent parameters to be specified in the constraint design. One benefit is that it approximately decouples the evolution of these angles from one phase to another; changing the evolution in one phase does not strongly affect the other as long as the boundary condition is maintained. This facilitates intuitively specifying 
the initial shape of the virtual constraints and makes the optimization task easier. For a list of independent parameters to be found by optimization, refer to Table IV in Appendix B.

For later use, we can organize the virtual constraints for each phase separately. For each $p \in \mathcal{P}$, we can define the output,

$$
y_{p}=h_{p}\left(q_{\mathrm{s}}, \alpha_{p}\right)=H_{0}^{p}\left(q_{\mathrm{s}}\right)-h_{d}^{p}\left(\theta_{\mathrm{s}}, \alpha_{p}\right),
$$

and,

$$
h_{d}^{p}\left(\theta_{\mathrm{s}}, \alpha_{p}\right)=\left[\begin{array}{cc}
h_{\mathrm{mLS}}^{d, p}\left(\theta_{\mathrm{s}}\right) \\
h_{\mathrm{mA}_{\mathrm{sw}}}^{d, p}\left(\theta_{\mathrm{s}}\right) \\
h_{\mathrm{mLS}}^{d, p}\left(\theta_{\mathrm{s}}\right) \\
h_{\mathrm{Tor}}^{d, p}\left(\theta_{\mathrm{s}}\right)
\end{array}\right] .
$$

The Bézier coefficients for each phase can be organized as,

$$
\alpha_{p}=\left[\begin{array}{c}
\alpha_{\mathrm{mLS}}^{p} \\
\alpha_{\mathrm{LA}}^{p} \\
\alpha_{\mathrm{mLS}}^{p} \\
\alpha_{\mathrm{Tor}}^{p}
\end{array}\right] .
$$

Remark 2: Both the local virtual constraint $h_{d}^{p}$ and the local selection of the controlled variables $H_{0}^{p}$ can be modified for each subphase resulting in (28). Here we only change the parameters used in the Bézier polynomials $\alpha_{v}^{p}$ and leave $H_{0}^{p}=H_{0}^{\mathrm{s}}$ as defined in (23), and $h_{d}^{p}=h_{d}^{\mathrm{s}}$ as defined in (24), for each phase $p \in \mathcal{P}$.

\section{B. Stance Zero Dynamics}

The organization of the stance phase into four subphases creates four continuous dynamics and discrete transitions between them. As discussed in Section III-A6, for each phase $p \in \mathcal{P}$, an output function $y_{p}$ has been associated with the continuous stance dynamics defined in (12). The zero dynamics is defined as the maximal internal dynamics of the system that is compatible with the output being identically zero (Isidori, 1995). Differentiating the output twice with respect to time results in

$$
\frac{d^{2} y_{p}}{d t^{2}}=L_{f_{\mathrm{s}}}^{2} h_{p}\left(x_{\mathrm{s}}, \alpha_{p}\right)+L_{g_{\mathrm{s}}} L_{f_{\mathrm{s}}} h_{p}\left(q_{\mathrm{s}}, \alpha_{p}\right) u
$$

where $L_{g_{\mathrm{s}}} L_{f_{\mathrm{s}}} h_{p}\left(q_{\mathrm{s}}, \alpha_{p}\right)$, the decoupling matrix, has full rank. Under the conditions of (Westervelt et al., 2007, Lemma 5.1),

$$
\begin{array}{r}
u^{*}\left(x_{\mathrm{s}}, \alpha_{p}\right):=-\left(L_{g_{\mathrm{s}}} L_{f_{\mathrm{s}}} h_{p}\left(q_{\mathrm{s}}, \alpha_{p}\right)\right)^{-1} \\
L_{f_{\mathrm{s}}}^{2} h_{p}\left(x_{\mathrm{s}}, \alpha_{p}\right),
\end{array}
$$

is the unique control input that renders the smooth fourdimensional embedded submanifold

$$
\begin{array}{r}
\mathcal{Z}_{\alpha_{p}}=\left\{x_{\mathrm{s}} \in T Q_{\mathrm{s}} \mid h_{p}\left(q_{\mathrm{s}}, \alpha_{p}\right)=0,\right. \\
\left.L_{f_{s}} h_{p}\left(x_{\mathrm{s}}, \alpha_{p}\right)=0\right\}
\end{array}
$$

invariant under the stance dynamics (12); that is, for every $z \in \mathcal{Z}_{\alpha_{p}}$,

$$
f_{p}^{*}(z):=f_{\mathrm{s}}(z)+g_{\mathrm{s}}(z) u^{*} \in T_{z} \mathcal{Z}_{\alpha_{p}} .
$$

Achieving the virtual constraints by zeroing the corresponding outputs reduces the dimension of the system by restricting its dynamics to the submanifold $\mathcal{Z}_{\alpha_{p}}$ embedded in the continuous-time state space $T Q_{\mathrm{s}} . \mathcal{Z}_{\alpha_{p}}$ is called the zero dynamics manifold and the restriction dynamics $\dot{z}=f_{p}^{*} \mid \mathcal{Z}_{\alpha_{p}}(z)$ is called the zero dynamics.

From Lagrangian dynamics (the derivation is standard (Westervelt et al., 2007, Chap. 5) and skipped for sake of brevity), a valid set of coordinates on $\mathcal{Z}_{\alpha_{p}}$ is

$$
x_{z d}^{p}=\left[\begin{array}{c}
\xi_{1} \\
\xi_{2} \\
\xi_{3} \\
\xi_{4}
\end{array}\right]=\left[\begin{array}{c}
\theta_{s} \\
q_{\mathrm{Bsp}_{\mathrm{st}}} \\
\frac{\partial \mathcal{L}_{s}}{\partial \dot{q}_{\mathrm{Bsp}}} \\
\frac{\partial \mathcal{L}_{s}}{\partial \dot{q}_{\mathrm{Tor}}}
\end{array}\right]
$$

This set of coordinates explicitly contains the $B_{\text {spring }}$ variable, which illustrates clearly that the zero dynamics is compliant:

$$
\dot{x}_{z d}^{p}=\left[\begin{array}{c}
\dot{\xi}_{1} \\
\dot{\xi}_{2} \\
\dot{\xi}_{3} \\
\dot{\xi}_{4}
\end{array}\right]=\left[\begin{array}{c}
L_{f_{s}} \theta_{s} \\
L_{f_{s}} q_{\mathrm{Bsp}_{\mathrm{st}}} \\
\frac{\partial \mathcal{L}_{s}}{\partial q_{\mathrm{Bsp}_{\mathrm{st}}}}+\tau_{s p} \\
\frac{\partial \mathcal{L}_{s}}{\partial q_{\mathrm{Tor}}}
\end{array}\right]
$$

\section{Event Transitions}

The division of the stance phase into subphases when specifying the virtual constraints in Section III-A necessitates the specification of the transition maps between the subphases. In preparation for the next section, we model the hybrid dynamics on the zero dynamics manifold by concatenating the solutions of the parameterdependent hybrid systems for each subphase

$$
\Sigma_{p}:\left\{\begin{array}{l}
x_{p} \in \mathcal{Z}_{\alpha_{p}} \\
\dot{x}_{p}=f_{p}^{*}\left(x_{p}\right) \\
\mathcal{S}_{p \rightarrow q}=\left\{x_{p} \in \mathcal{Z}_{\alpha_{p}} \mid H_{p \rightarrow q}\left(x_{p}\right)=0\right\} \\
x_{q}^{+}=\Delta_{p \rightarrow q}\left(x_{p}^{-}\right) .
\end{array}\right.
$$

The model captures the continuous-time dynamics of the system in phase $p \in \mathcal{P}$ and the discrete transition to phase $q \in \mathcal{P}$, with the only valid choice of transitions for walking being $(p, q) \in$ $\{(m c, s c),(s c, s i),(s i, s d),(s d, m c)\}$.

The switching surfaces, $\mathcal{S}_{p \rightarrow q}$, for the transitions for walking are defined by the zero level sets of the corresponding threshold functions $H_{p \rightarrow q}: T Q_{\mathrm{s}} \rightarrow \mathbb{R}$, which are given below,

$$
\begin{aligned}
H_{m c \rightarrow s c} & :=\theta_{\mathrm{s}}-\theta_{m c}^{-} \\
H_{s c \rightarrow s i} & :=q_{\mathrm{Bsp}_{\mathrm{st}}}-5^{\circ} \\
H_{s i \rightarrow s d} & :=\theta_{\mathrm{s}}-\theta_{s i}^{-} \\
H_{s d \rightarrow m c} & :=p_{\text {toe }_{\mathrm{sw}}}^{v}
\end{aligned}
$$

The transition maps, $\Delta_{p \rightarrow q}: S_{p \rightarrow q} \rightarrow T Q_{\mathrm{s}}$, provide the initial conditions for the ensuing phase $q \in \mathcal{P}$, and are 


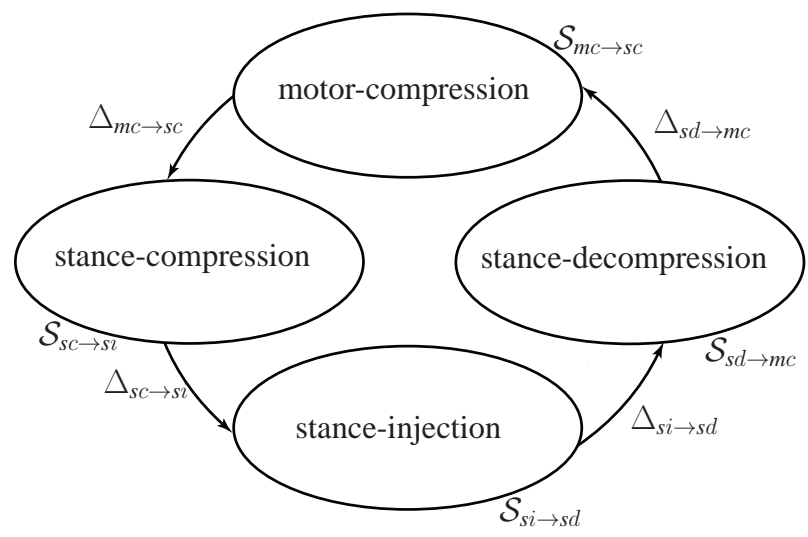

Fig. 4. The hybrid system for stance with continuous-time phases and discrete event transitions.

given below,

$$
\begin{aligned}
\Delta_{m c \rightarrow s c} & :=\mathrm{i} d \\
\Delta_{s c \rightarrow s i} & :=\mathrm{i} d \\
\Delta_{s i \rightarrow s d} & :=\mathrm{i} d \\
\Delta_{s d \rightarrow m c} & :=\Delta_{\mathrm{s} \rightarrow \mathrm{s}},
\end{aligned}
$$

where $\mathrm{i} d$ is the identity map and $\Delta_{\mathrm{s} \rightarrow \mathrm{s}}$ is defined in (14).

The event transitions are indicated in Figure 4. To find a set of values for the independent parameters of the constraint design specified in Section III-A, we employ the above hybrid system and formulate the problem as a constrained optimization.

\section{Gait Design Through Optimization}

A periodic walking gait is designed by selecting the free parameters in the virtual constraints. As in (Westervelt et al., 2003, 2007), this is most easily done by posing an optimization problem, such as minimum energy per step length, subject to constraints to meet periodicity, workspace and actuator limitations, and desired walking speed. The equations of the compliant zero dynamics, which are of reduced dimension compared to the full dynamics, are employed in the optimization for efficiency of computation.

The nonlinear constrained optimization routine fmincon of MATLAB's Optimization Toolbox is used to perform the numerical search for desired gaits. The quantities involved in optimization are the scalar cost function to be minimized, $J$, the vector of equality constraints, $\mathrm{EQ}$, and the vector of inequality constraints, INEQ. The optimization algorithm, equality and inequality conditions are given in Appendix B and the list of optimization parameters is specified in Table IV.

Several popular cost functions for bipedal gait design are given in (Westervelt et al., 2007, Sec. 6.3.3). Here, two cost criteria are used in in the optimization process. First we use a nominal cost function, as used in RABBIT in (Westervelt et al., 2004), consisting of the integral over a step of squared torque divided by distance traveled,

$$
J_{\text {nom }}\left(\alpha_{\mathrm{s}}\right)=\frac{1}{p_{\text {toe }_{\mathrm{sw}}}^{h}\left(q_{\mathrm{s}}^{-}\right)} \int_{0}^{T_{I}}\|u(t)\|^{2} d t,
$$

where $T_{I}$ is the step duration and $p_{\text {toe }_{\mathrm{sw}}}^{h}$ is the step length. Minimizing this cost function tends to reduce peak torque demands and minimizes the electrical energy consumed per step.

Next we use a cost function that quantifies the mechanical energy consumed. The specific mechanical cost of transport, $c_{m t}$ is introduced in (Collins et al., 2005; Collins and Ruina, 2005) as means of quantifying energy consumed for bipedal locomotion. $c_{m t}$ is the energy consumed per unit weight per unit distance traveled and can be defined as a cost function,

$$
J_{c_{m t}}=\frac{\int_{0}^{T_{I}} \sum_{i=1}^{4} E_{i}(t) d t}{M g d},
$$

where $M$ is the mass of the robot, $g$ is the acceleration due to gravity, $d$ is the distance traveled, and

$$
E_{i}(t)= \begin{cases}u_{i}(t) \dot{q}_{i}(t) & u_{i}(t) \dot{q}_{i}(t)>0 \\ 0 & u_{i}(t) \dot{q}_{i}(t) \leq 0\end{cases}
$$

Mechanical power can be either positive (energy is injected) or negative (energy is absorbed). Some authors, (Hobbelen and Wisse, 2008), consider the absolute mechanical power while defining the $c_{m t}$, whereas the definition in (40), (41) does not take into account any negative work that is performed by the actuators, the idea being that if the actuators were redesigned, energy could be absorbed mechanically through a friction brake or electronically through regenerative breaking.

\section{E. Fixed Point for Walking}

This section presents a nominal fixed point of $0.8 \mathrm{~m} / \mathrm{s}$ obtained by applying the optimization procedure outlined in Section III-D to the virtual constraints of Section III-A, and with the cost function (39). Figure 5 illustrates the nominal evolution of the virtual constraints and other configuration variables for one step. It is seen that the stance motor leg shape is held constant for the first part of the gait right after impact, and both the stance motor leg shape and the torso are held constant towards the final part of the gait. Interestingly, the torso moves less than two degrees throughout the step.

Figure 6 illustrates the evolution of the leg shape and the stance $B_{\text {spring variables. Notice that the spring }}$ compresses to its peak value, and the $s c \rightarrow$ si transition is triggered as the spring decompresses to five degrees. The injection of energy in the si-phase causes the spring to compress again. Figure 7 illustrates the actuator torques used to realize the gait. These torques are small in comparison to the peak torque capacities of the actuators: $30 \mathrm{Nm}$ at $u_{\mathrm{mLA}}$ and $55 \mathrm{Nm}$ at $u_{\mathrm{mLS}}$. The torques are discontinuous at phase boundaries, as noted earlier, due to the choice of the virtual constraints being $\mathcal{C}^{1}$ at phase boundaries. Figure 8 illustrates the evolution 


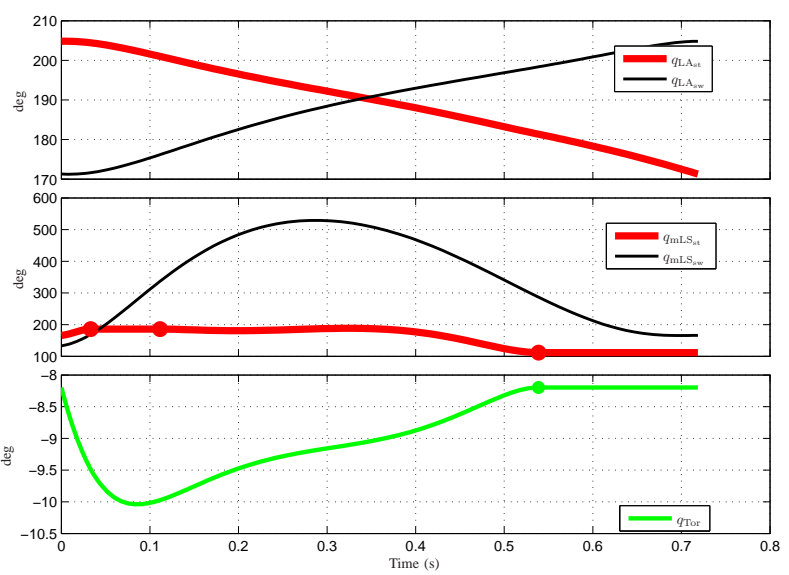

Fig. 5. Evolution of the virtual constraints and configuration variables for a nominal fixed point (periodic walking gait) at a speed of 0.8 $\mathrm{m} / \mathrm{s}$ and step length $0.575 \mathrm{~m}$. The dots on the stance motor legshape virtual constraint illustrate the location of transition between consecutive subphases.

of the swing leg height and the vertical position of the center of mass (COM) of the robot. The COM moves downward immediately after impact, before reversing course and following a roughly parabolic path. Such a trajectory more closely resembles that of a human (Lee and Farley, 1998) than that of a robot with rigid links and rigid gearing. The specific cost of mechanical transport for this nominal gait is, $c_{m t}=0.0452$. The corresponding power plot is very similar to the power plot for the next designed gait and is not shown.

A second walking gait was designed, this time using cost function (40), which optimizes for the specific cost of mechanical transport. The optimization terminated with a value of $c_{m t}=0.0385$ which is over $10 \%$ lower than that for the nominal gait. For this fixed point, Figure 9 compares the total power provided by the stance leg shape motor to the total power at stance leg shape, where the latter is the sum of the actuator and compliance power. It is clear that the spring is doing the vast majority of the negative work that is necessary on the stance leg.

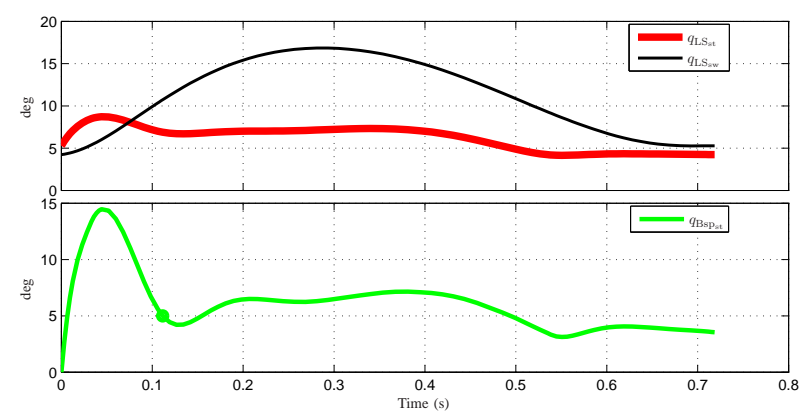

Fig. 6. Evolution of the leg shape and stance $B_{\text {spring variables }}$ corresponding to the nominal fixed point. The dot on the $\mathrm{B}_{\text {spring }}$ plot illustrates the location of the $s c$ to $s i$ event transition and corresponds to $q_{\mathrm{Bsp}_{\mathrm{st}}}=5^{\circ}$.

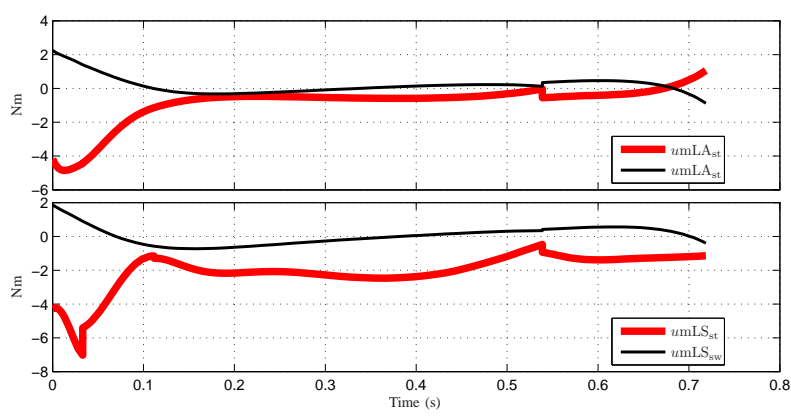

Fig. 7. Actuator torques corresponding to the nominal fixed point. Note that the torques are discontinuous at subphase boundaries, due to the choice of $\mathcal{C}^{1}$ continuity of the virtual constraints at subphase boundaries as per Appendix A-A.

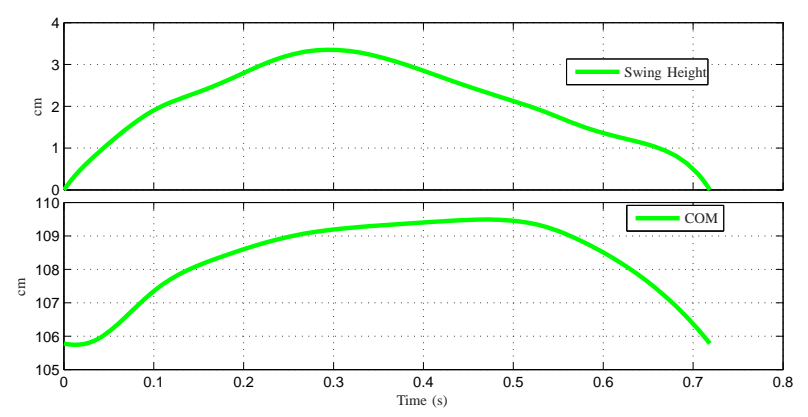

Fig. 8. Evolution of swing leg height and vertical center of mass (COM) of the robot for the nominal fixed point. The COM trajectory more closely resembles that of a human gait than of a robot with rigid links since the COM moves downward immediately after impact, before reversing course and following a roughly parabolic path.

\section{Closed-loop Design AND Stability ANALYSIS}

The feedback presented in (32) renders the zero dynamics manifold invariant under the stance phase dynamics. It is used in the optimization process of gait design in order to evaluate the torques along a solution of the model respecting the virtual constraints. The feedback (32) does not however render the solution stable or attractive in any way. In the following, two controllers

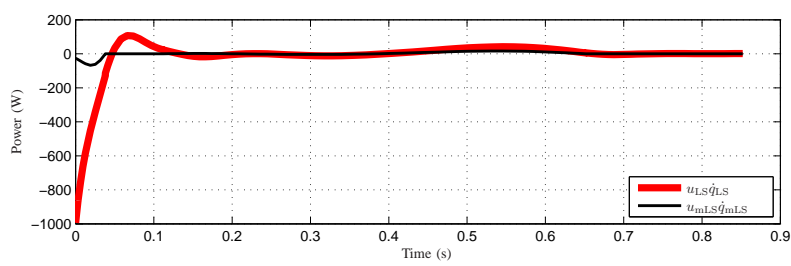

Fig. 9. Power plot of a fixed point obtained by optimization of (40), specific cost of mechanical transport. The thick line illustrates the total instantaneous power at the leg shape from the actuator and the spring, and the thin line illustrates the instantaneous power at the leg shape from the motor alone. The difference is the energy that is saved and that would otherwise have to be provided by the actuator if the spring were absent. This plot shows the significant energy economy at impacts due to the presence of the compliance in the transmission. 
based on the classic input-output linearizing controller

$$
\begin{array}{r}
u=u^{*}\left(x_{\mathrm{s}}, \alpha_{p}\right)-L_{g_{\mathrm{s}}} L_{f_{\mathrm{s}}} h_{p}\left(q_{\mathrm{s}}, \alpha_{p}\right)^{-1} \\
\left(\frac{K_{p, P}}{\epsilon^{2}} y+\frac{K_{p, D}}{\epsilon} \dot{y}\right),
\end{array}
$$

where $p \in\{m c, s c, s i, s d\}$, are discussed.

\section{A. A PD + Feedforward Controller}

With an eye toward experimental implementation, we look at successful controllers that have been employed to enforce virtual constraints in experiments. For RABBIT, it was possible to implement the virtual constraints through a simple PD controller (Westervelt et al., 2004), per

$$
u=-K_{P} y-K_{D} \dot{y}
$$

for $y$ given by (22), and $\dot{y}$ computed numerically. On MABEL, such a controller (employed in experiments in (Grizzle et al., 2009)) resulted in virtual constraints that were not accurately achieved due to large tracking errors, and attempts at reducing the errors with high controller gains were unsuccessful. See Section VI-F for discussion of this point.

To address this, the vector of nominal control torques $u^{*}$ from (32) is incorporated as a feedforward term in the PD controller. In particular, along the nominal orbit, for each of the actuated variables $m \in \mathcal{M}$ and for each of the phases $p \in \mathcal{P}, u^{*}$ is regressed against $\theta_{\mathrm{S}}$ with $5^{\text {th }}$ order Bézier polynomials to obtain the Bézier coefficients $\beta_{p}^{m}$ and resulting in the controller

$$
u_{e x p}=u^{*}\left(\theta_{\mathrm{s}}, \beta_{\mathrm{s}}\right)-K_{P} y-K_{D} \dot{y},
$$

where, $y$ is as defined in (22), $\dot{y}$ still computed numerically, and $\beta_{\mathrm{s}}=\left(\beta_{p}^{m}\right)$.

The stability of the fixed-points with the proposed closed-loop controller (43) can be tested numerically using a Poincaré map $P: \mathcal{S} \rightarrow \mathcal{S}$ with the switching surface taken to be the switching surface at the $s i \rightarrow s d$ event transition, i.e., $\mathcal{S}=\mathcal{S}_{s i \rightarrow s d}$, and

$$
P\left(x_{\mathrm{s}}\right)=\phi\left(T_{I} \circ \Delta_{s i \rightarrow s d}\left(x_{\mathrm{s}}\right), \Delta_{s i \rightarrow s d}\left(x_{\mathrm{s}}\right)\right),
$$

where, $\phi\left(t, x_{0}\right)$ denotes the maximal solution of (12), with initial condition $x_{0}$ at time $t_{0}=0$ and with $u$ as defined in (43). Finally, $T_{I}$ is the time-to-impact function defined in the usual way (Westervelt et al., 2007, p. 94).

Using the Poincaré return map (44), we can numerically calculate the eigenvalues of its linearization about the fixed-point. The analysis shows that the walking gait obtained by optimizing (39) and with the closed-loop controller (43) is exponentially stable with a dominant eigenvalue of 0.6921 .

Similarly, the gait obtained from optimizing (40) is also exponentially stable, with a dominant eigenvalue of 0.8194 .

\section{B. Hybrid Invariance}

The above controllers are not hybrid invariant. It was discovered in (Morris and Grizzle, 2006, 2009) that, in the presence of compliance, while the feedback controller (42) will render the zero dynamics manifold of a given phase invariant under the continuous dynamics, it will not necessarily render it invariant under the transition maps, that is, at transitions from one phase to another, invariance is lost. The loss of invariance manifests itself as an impulsive disturbance to the control law at each transition off the periodic orbit. These perturbations do not prevent asymptotic stability from being achieved, but they do cause the actuators to do more work. The reference (Morris and Grizzle, 2009) proposed a supplemental event-based controller that eliminates this issue and, in fact, creates a hybrid zero dynamics for the closed-loop system, that is, the zero dynamics manifold is invariant under the continuous dynamics as well as the transition maps.

For the related robot, Thumper, (Poulakakis, 2008; Poulakakis and Grizzle, 2009a) propose an event-based control at each phase transition. This is not practical here, however, because we have certain phases with extremely small duration (the $m c$ phase for instance). Instead, we create a hybrid zero dynamics by updating parameters only at the impact event (swing leg contacts the ground).

Following (Morris and Grizzle, 2009; Grizzle et al., 2008), the virtual constraints are modified stride to stride so that they are compatible with the initial state of the robot at the beginning of each step. The new output for the feedback control design is,

$$
\begin{aligned}
y_{c} & =h_{\mathrm{s}}\left(q_{\mathrm{s}}, y^{s+}, \dot{y}^{s+}\right) \\
& =H_{0}^{\mathrm{s}} q_{\mathrm{s}}-h_{d}^{\mathrm{s}}\left(\theta_{\mathrm{s}}\right)-h_{c}^{\mathrm{s}}\left(\theta_{\mathrm{s}}, y^{s+}, \dot{y}^{s+}\right) .
\end{aligned}
$$

The output consists of the previous output, (22), and an additional correction term that depends on the previous output evaluated at the beginning of the step, specifically, $y^{s+}=H_{0}^{\mathrm{s}} q_{\mathrm{s}}^{+}-h_{d}^{\mathrm{s}}\left(\theta_{\mathrm{s}}^{+}\right)$, and $\dot{y}^{s+}=H_{0}^{\mathrm{s}} \dot{q}_{\mathrm{s}}^{+}-\frac{\partial h_{d}\left(\theta_{\mathrm{s}}\right)}{\partial \theta_{\mathrm{s}}} \dot{\theta}_{\mathrm{s}}^{+}$. The values of $y^{s+}$, and $\dot{y}^{s+}$ are determined at the beginning of each step and held constant throughout the step. The function $h_{c}^{\mathrm{s}}$ is taken here as

$$
h_{c}^{\mathrm{s}}\left(\theta_{\mathrm{s}}\right)=\left[\begin{array}{c}
0 \\
h_{\mathrm{LA}_{\mathrm{sw}}}^{c}\left(\theta_{\mathrm{s}}\right) \\
h_{\mathrm{mLS}}^{c}\left(\theta_{\mathrm{s}}\right) \\
h_{\text {Tor }}^{c}\left(\theta_{\mathrm{s}}\right)
\end{array}\right],
$$

with each $h_{v}^{c}\left(\theta_{\mathrm{s}}\right), v \in \mathcal{V} \backslash\left\{\mathrm{mLS}_{\mathrm{st}}\right\}$ taken to be twice continuously differentiable functions of $\theta_{\mathrm{s}}$ such that,

$$
\left\{\begin{array}{ll}
h_{v}^{c}\left(\theta_{\mathrm{s}}, y^{s+}, \dot{y}^{s+}\right) & =y^{s+} \\
\frac{\partial h_{v}^{c}}{\partial \theta_{s}}\left(\theta_{\mathrm{s}}^{+}\right) & =\frac{\dot{y}^{s+}}{\dot{\theta}_{s}^{+}} \\
h_{v}^{c}\left(\theta_{\mathrm{s}}, y^{s+}, \dot{y}^{s+}\right) & =0, \frac{\theta_{\mathrm{s}}^{+}+\theta_{v}^{m c+}}{2} \leq \theta_{\mathrm{s}} \leq \theta_{v}^{m c-}
\end{array} .\right.
$$

With $h_{v}^{c}$ designed this way, the initial errors of the output and its derivative are smoothly joined to the original virtual constraint at the middle of the first phase of 
the corresponding virtual constraint. By the choice of $\theta_{\mathrm{LA}}^{m c-}, \theta_{\mathrm{mLS}}^{m c-}$ and $\theta_{\mathrm{Tor}}^{m c-}$ (defined in Section III-A), the joining of the swing leg virtual constraints occurs at the middle of the step, while the joining for the torso virtual constraint occurs earlier, at the middle of the combined phases $m$ cscsi. This is illustrated in Figure 3 with thick dashed lines.

As noted in the definition of $h_{c}^{\mathrm{s}}$ in (46), we have selected $h_{\mathrm{mLS}_{\mathrm{st}}}^{c} \equiv 0$ since the $m \mathrm{c}$ phase is too short to handle significant transients without large actuator torques, and further we want to enforce the virtual constraint in the sc phase to be constant in order to effectively use the compliance. To overcome this, we propose an event-based control action specific for the $\mathrm{mLS}_{\mathrm{st}}$ virtual constraint that updates $\alpha_{\mathrm{mLS}}^{m c}, \alpha_{\mathrm{mLS}}^{s c}, \alpha_{\mathrm{mLS}}^{s i}$ at the beginning of each step such that during the $m \mathrm{c}$ phase, the virtual constraint only drives the motor leg shape velocity to zero, and during the $s c$ phase, the virtual constraint keeps the motor shaft locked at a constant position. Not until the si phase does the modified virtual constraint smoothly join the nominal virtual constraint. This correction term is also illustrated in Figure 3 with thick dashed lines.

Under the new control law defined by (45), the behavior of the robot is completely defined by the event transition maps and the swing phase zero dynamics, with $h_{d}^{\mathrm{s}}$ replaced by $h_{d}^{\mathrm{s}}+h_{c}^{\mathrm{s}}$. The stability of the fixed-point $x^{*}$ can now be tested numerically using a restricted Poincaré map $\rho: \mathcal{S} \cap \mathcal{Z} \rightarrow \mathcal{S} \cap \mathcal{Z}$ where $\mathcal{Z}=\left\{x_{\mathrm{s}} \in T Q_{\mathrm{s}} \mid y_{c}\left(q_{\mathrm{s}}\right)=0, \dot{y}_{c}\left(q_{\mathrm{s}}\right)=0\right\}$, the switching surface is taken to be the switching surface at the $s i \rightarrow s d$ event transition, i.e., $\mathcal{S}=\mathcal{S}_{s i \rightarrow s d}$, and

$$
\rho\left(x_{\mathrm{s}}\right)=\phi\left(T_{I} \circ \Delta_{s i \rightarrow s d}\left(x_{\mathrm{s}}\right), \Delta_{s i \rightarrow s d}\left(x_{\mathrm{s}}\right)\right),
$$

where, $\phi\left(t, x_{0}\right)$ denotes the maximal solution of (12), with initial condition $x_{0}$ at time $t_{0}=0$ and $u$ as defined in (42). Hybrid invariance is achieved because the transition map for these events, $\Delta_{s i \rightarrow s d}$ is the identity map, and $\Delta_{s i \rightarrow s d}\left(\mathcal{S}_{s i \rightarrow s d} \cap \mathcal{Z}\right) \subset \mathcal{S}_{s i \rightarrow s d} \cap \mathcal{Z}$.

Using the restricted Poincaré return map (48), we can numerically calculate the eigenvalues of its linearization about the fixed-point. For the gait obtained by optimizing (39), we obtain the eigenvalues

$$
\operatorname{eig}\left(\frac{\partial \rho\left(x_{\mathrm{s}}\right)}{\partial x_{\mathrm{s}}}\right)=\left[\begin{array}{r}
0.7258 \\
2.6380 e-5 \\
-1.8001 e-6
\end{array}\right] \text {. }
$$

From (Morris and Grizzle, 2009, Cor. 2), the feedback (42) and (45) renders the periodic orbit of the closedloop system exponentially stable for $\epsilon$ in (42) sufficiently small, and $K_{P}, K_{D}$ such that $\lambda^{2}+K_{D} \lambda+K_{P}=0$ is Hurwitz.

The orbit obtained from optimizing (40) is also exponentially stable, with a dominant eigenvalue 0.7065 .

\section{EXPERIMENTS}

This section documents experimental implementations of the controllers of Section IV in various walking scenarios. Figure 10 depicts the experimental setup. To illustrate the power and limitations of the proposed methods, five experiments are presented. First, the robustness and efficiency of walking motions, resulting from enforcing the virtual constraints of Section III through a feedforward plus PD controller developed in Section IV-A, is evaluated. Then, to achieve fast walking motions, the full compliant HZD controller developed in Section IV-B is implemented.

For each experiment, the controller was first coded in $\mathrm{C}++$ and evaluated on a detailed simulation model of the robot that included encoder quantization and numerical estimation of velocity variables from encoder measurements. The controller was tested under various model perturbations, such as errors in the torso mass, spring stiffness, torso center of mass position, and deviations in initial conditions. These simulations are not discussed here for the sake of brevity. The simulation model was then replaced with the physical robot. The experimental protocol is identical to the one used in (Westervelt et al., 2004, Sect. 4). The experiments varied in duration from 78 steps to 265 steps, and were ended in each case by the experimenter stopping the robot and killing the power.

The results of the experiments are presented in Figures 11-19. In order to facilitate comparisons, Figures 11-13 assemble results from Exp. 1, 4 and 5; the remaining figures pertain to individual experiments. In the experiments, the left leg refers to the inner leg, which is closer to the center boom, and the right leg refers to the outer leg, which is farther from the center boom. All walking speeds are measured with respect to the center point of the hip between the two legs. Videos of the experiments are available on YouTube (Grizzle, 2010a).

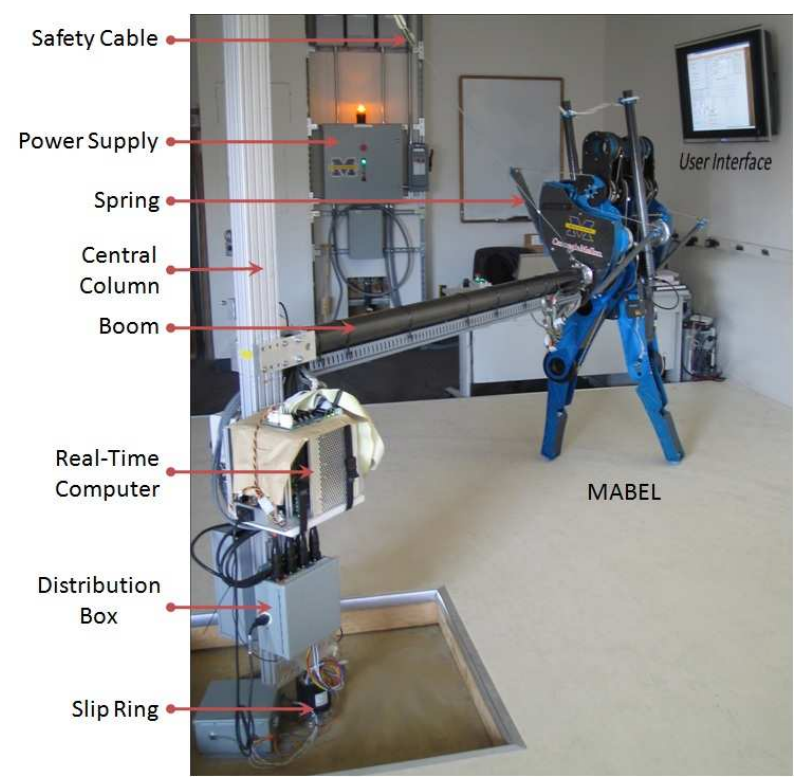

Fig. 10. Experimental setup of the bipedal testbed MABEL. 


\section{A. Exp. 1: Nominal Walking at a Fixed Speed}

This experiment approximately implements the virtual constraints depicted in Figure 5 with the feedforward plus PD controller given in (43). It was noticed in early experiments that the transition from the $s \mathrm{c}$ phase to the si phase given by (37) did not always occur. The spring was not decompressing to the $5^{\circ}$ trigger point, and was probably due to the initial few steps being far away from the nominal orbit, and also because of inability of the controller to accurately track the stance motor leg shape virtual constraint. To ensure that the transition from the sc phase to the si phase always occurred in the experiments, the switching surface for this transition is modified to have a guard around the nominal value of $\theta_{s c}^{-}$, such that the transition is guaranteed to occur for $\theta_{\mathrm{s}} \in\left(\theta_{s c}^{-}-\gamma, \theta_{s c}^{-}+\gamma\right]$, with $\gamma$ being a small positive quantity. The modified switching surface is

$$
\begin{aligned}
& \mathcal{S}_{s c \rightarrow s i}^{\exp }:=\left(\mathcal { S } _ { s c \rightarrow s i } \cap \left\{x_{\mathrm{s}} \in T Q_{\mathrm{s}} \mid \theta_{\mathrm{s}} \in\right.\right. \\
& \left.\left.\left(\theta_{s c}^{-}-\gamma, \theta_{s c}^{-}+\gamma\right]\right\}\right) \cup \\
& \left\{x_{\mathrm{s}} \in T Q_{\mathrm{s}} \mid \theta_{\mathrm{s}}=\theta_{s c}^{-}+\gamma\right\}
\end{aligned}
$$

This controller led to successful walking, as illustrated in Figures 11 through 15. The evolution of the desired and achieved virtual constraints is depicted for the swing leg variables in Figure 11(a) and for the torso and stance leg motor positions in Figure 12(a). The nominal tracking in the swing leg is very good, whereas considerable errors occur in the torso and stance leg motor position. This is consistent with the fact that the swing leg is unloaded and lightweight, and hence much easier to control. The torques are given in Figure 13(a).

Figure 14 depicts the evolution of the torso angle and the evolution of $\mathrm{B}_{\text {spring }}$ for the left and right legs in stance, respectively, over 52 of the 78 steps in the experiment. Each of these is compared to the nominal fixed point. There is a pronounced asymmetry in the robot, as was noted in (Grizzle et al., 2009). This asymmetry is due to the boom radius not being large enough and is currently not included as part of the model. It is also evident that the experimental gait is faster than the designed value: the nominal fixed point is $0.8 \mathrm{~m} / \mathrm{s}$, whereas the average experimental speed is approximately $1.0 \mathrm{~m} / \mathrm{s}$. One possible reason for the speed discrepancy is the impact model; see (Westervelt et al., 2004, p. 569). This is discussed in Section VI. Another possible reason is the large errors in tracking the virtual constraints. As will be seen, controllers in subsequent experiments will reduce these errors and the walking speed will be closer to that of the fixed point.

Finally, Figure 15 illustrates the evolution of the

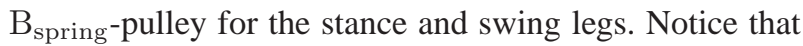
the $s c \rightarrow s i$ transition does not occur at five degrees as on the nominal orbit, and that in the swing phase, $\mathrm{B}_{\text {spring }}$ is not fixed at zero as assumed in the model.

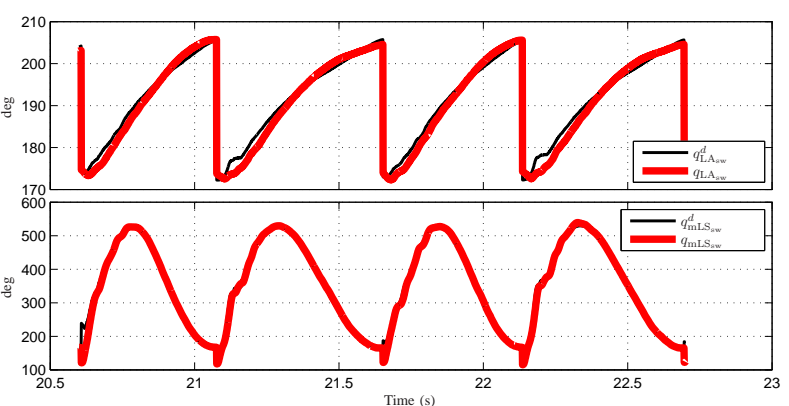

(a)

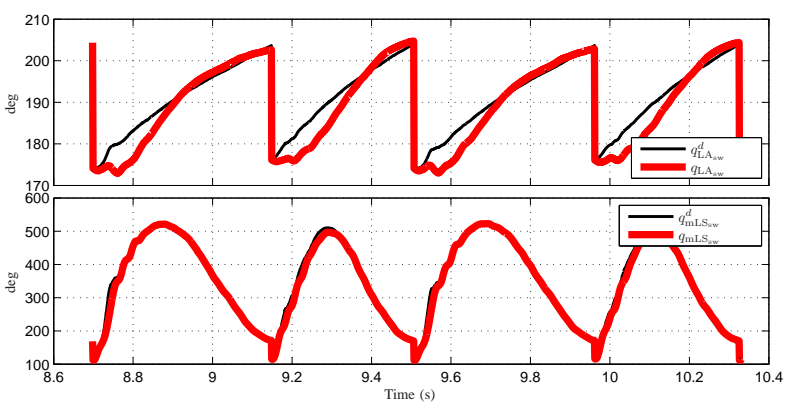

(b)

Fig. 11. Tracking for the swing-leg virtual constraints for (a) PD + Feedforward controller in Exp. 1, and (b) Decentralized zero dynamics controller in Exp. 5. The tracking for PD + Feedforward controller is quite good, whereas the tracking for decentralized zero dynamics controller is worse right after impact and recovers quite well near impact.

\section{B. Exp. 2: Demonstration of Robustness to Perturbations}

To test the robustness of the controller used in Exp. 1, external, short duration forces are applied at the hip at various instants of time. The results are depicted in Figure 16. Initially, the robot is pushed forward by the experimenter, causing the robot to speed up by roughly $45 \%$. During the subsequent ten steps, the speed slowly converges back to the nominal. Next, a retarding force is applied, causing the robot to slow down by roughly $45 \%$. Over the next three steps, the robot's speed has essentially returned to the unperturbed value. This experiment demonstrates the robustness of the robot in closed loop with the feedback controller given by (43) to external disturbances and illustrates an asymmetry in the rejection of the speed perturbation. A simulation of the model was carried out to estimate the force applied. A constant force over the second half of the gait was assumed to be applied at the end of the boom. In order to achieve similar speed gains and speed drops, the required force from simulation is around $78 \mathrm{~N}$ in the forward direction, and around $71 \mathrm{~N}$ in the reverse direction, respectively.

When the robot is pushed forward (external energy is injected into the system), the speed of the robot increases, and the robot takes a large number of steps to recover. One would expect that, due to the increased speed, larger amounts of energy would be dissipated at impacts after the forward push perturbation. This 


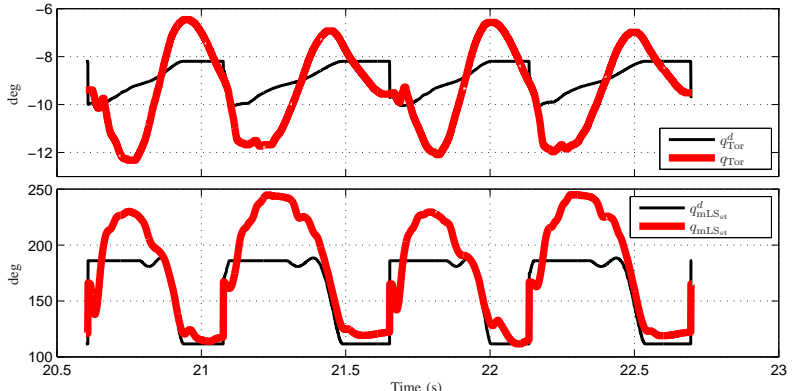

(a)

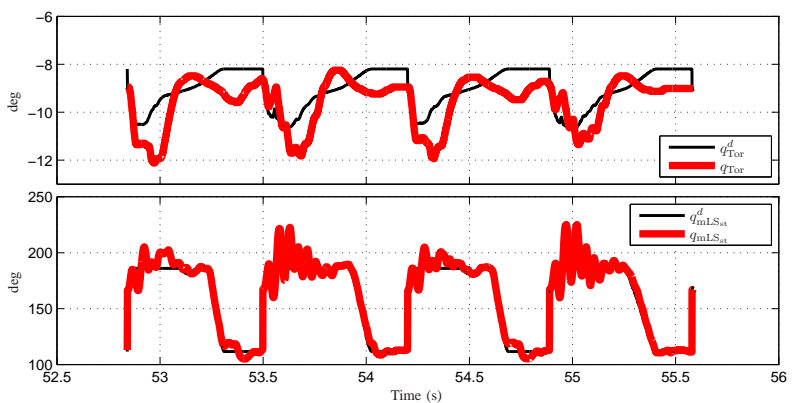

(b)

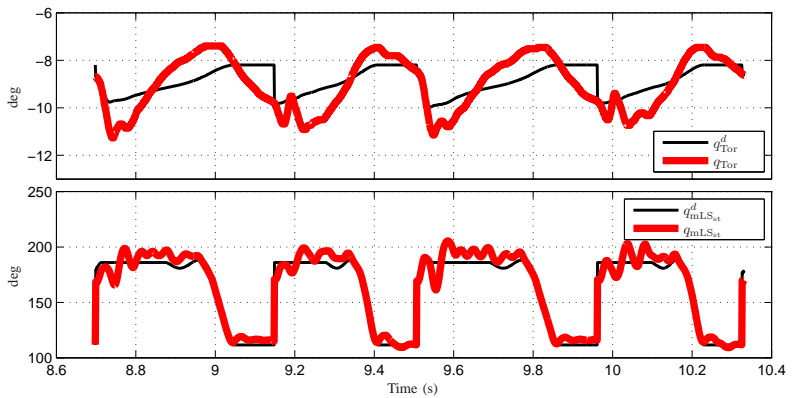

(c)

Fig. 12. Tracking for the stance-leg virtual constraints for (a) PD + Feedforward controller in Exp. 1, (b) Compliant zero dynamics controller in Exp. 4, and (c) Decentralized zero dynamics controller in Exp. 5. The PD + Feedforward controller produces significant tracking errors. The tracking fidelity is much improved using the compliant zero dynamics controller. However, there are significant oscillations in tracking the motor leg shape, corresponding to a peak variation of approximately $1.3^{\circ}$ in $q_{\mathrm{LS}}$. The decentralized zero dynamics controller improves the tracking even further, with the oscillations nearly eliminated.

would cause the robot to converge to its nominal motion faster. However, this is not the case in the experiment, indicating that the energy loss at impact is fairly small. Thus a large number of steps are needed to dissipate the extra energy injected into the system by the push. This is also what we see in simulation in Section VI-B. This indicates that the compliance plays an important role in the impacts. For pushes in the opposite direction, additional energy is quickly injected into the system by the actuators.

\section{Exp. 3: Efficient Walking}

The fixed point obtained by optimizing for the specific energetic cost of mechanical transport (40) is imple-

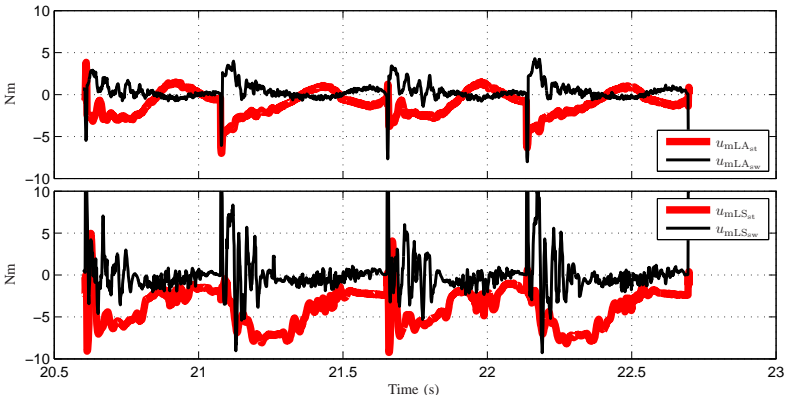

(a)

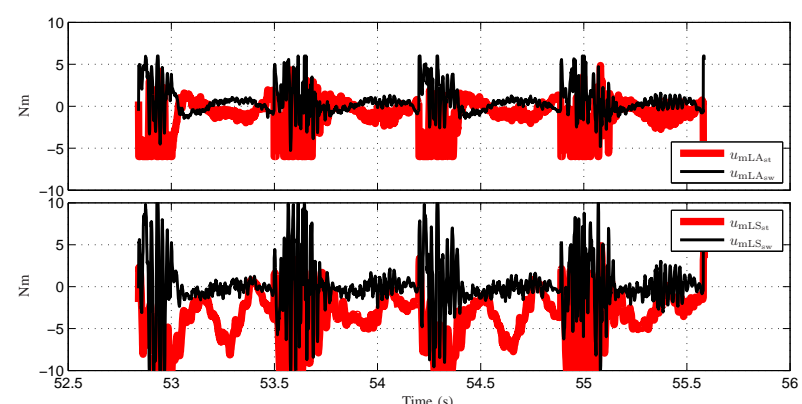

(b)

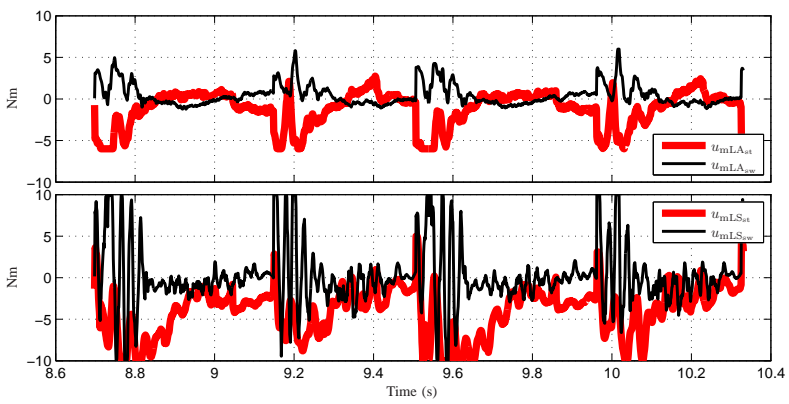

(c)

Fig. 13. Motor torques for (a) PD + Feedforward controller in Exp. 1, (b) Compliant zero dynamics controller in Exp. 4, and (c) Decentralized zero dynamics controller in Exp. 5. The torques for the PD + Feedforward controller are noisy, but are comparable in magnitude to the nominal predicted values presented in Figure 7. The torques for the compliant zero dynamics controller appear more 'noisy' and experience significant saturation (saturation limits were set to 6 $\mathrm{Nm}$ on leg angle motors and $10 \mathrm{Nm}$ on leg-shape motors). For the decentralized zero dynamics controller, the leg angle motor torques are far less 'noisy' than those for the compliant zero dynamics controller, while the leg shape motor torques are still a little 'noisy', but still much reduced when compared to the compliant zero dynamics controller.

mented experimentally using the feedforward plus PD controller (43). The designed fixed point has a cost of $c_{m t}=0.0385$ and nominal walking speed of $0.8 \mathrm{~m} / \mathrm{s}$.

Figure 17 depicts the power plots for the inside and outside legs obtained by averaging the experimental data over 77 steps. The realized energetic cost of mechanical transport is $c_{m t}=0.14^{3}$. For comparison purposes, Figure 18 shows the power plot for the hand-tuned virtual constraints reported in (Grizzle et al., 2009). It

${ }^{3}$ This is around $10 \%$ lower than the realized energetic cost of mechanical transport for Exp. 1, which was $c_{m t}=0.15$. The designed values of the energetic cost of mechanical transport for the corresponding fixed points also differ by the same amount. 

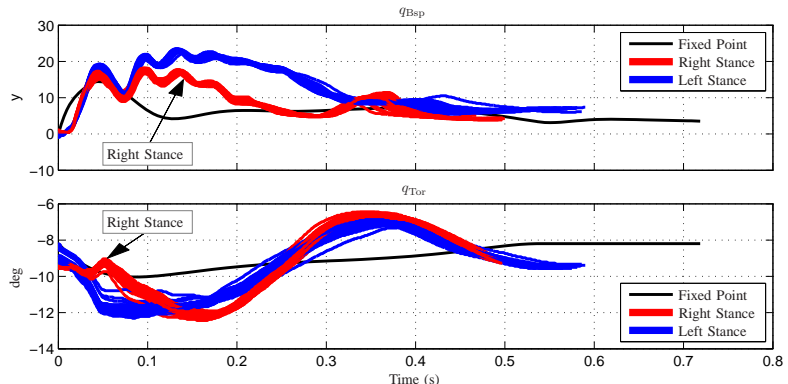

Fig. 14. This figure compares the evolution of $q_{\mathrm{Bsp}}$ for the left and right legs in stance for Exp. 1 and the evolution of the torso, over 52 steps, and compares them with the corresponding values for the nominal fixed point. The step times for the left and right legs vary, with the robot walking faster when the right (i.e., inside) leg is stance, and both step times are shorter than the fixed point. This is evident when we compare the average walking speed in the experiment, 1.0 $\mathrm{m} / \mathrm{s}$, with the designed fixed point walking speed of $0.8 \mathrm{~m} / \mathrm{s}$. Possible reasons for this discrepancy are discussed in the text. Next notice in the $q_{\text {Bsp }}$ plot, right after impact, in the experiments the spring compresses more slowly than in the fixed point. This is possibly due to a noninstantaneous double support phase in the experiments.

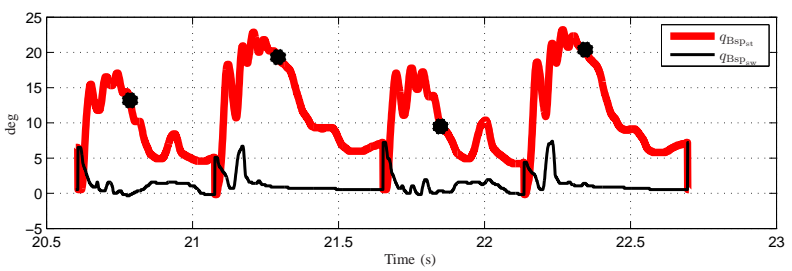

Fig. 15. Stance and swing $B_{\text {spring }}$ evolution for nominal experiment. The asterisks indicate the locations of the transitions from subphase $s c$ to subphase $s i$. This transition occurs before the $q_{\mathrm{Bsp}}=5^{\circ}$ event due to the presence of the additional guards in the experiments, (50). It is also seen that the swing $\mathrm{B}_{\text {spring }}$ angle is not at $0^{\circ}$ as assumed in the model. This deviation is significant right after impact, but appears to quickly die out to a small value for the remaining part of the gait.

is clear that the new control design makes more efficient use of the compliance available in the open-loop plant than the controllers previously designed.

The experimentally realized $c_{m t}$ is approximately three times the designed value. This is discussed in Section VI-C.

\section{Exp. 4: Compliant Zero Dynamics Controller}

This section focuses on achieving greater fidelity in the tracking of the virtual constraints.

Background: An important goal of MABEL is running. As an intermediate goal, we have started looking at fast walking. We consequently designed new fixed points using the methods of Sections III and III-D, for walking at 1.0 and $1.2 \mathrm{~m} / \mathrm{s}$, and implemented them using the controller structure of (43). Experiments were unsuccessful, even when the transition controller of (Westervelt et al., 2007, Chap. 7) was added. The experimental data showed poor tracking of the torso and stance motor leg shape virtual constraints and led us to conclude that more precise control was needed in order to achieve these higher speeds.

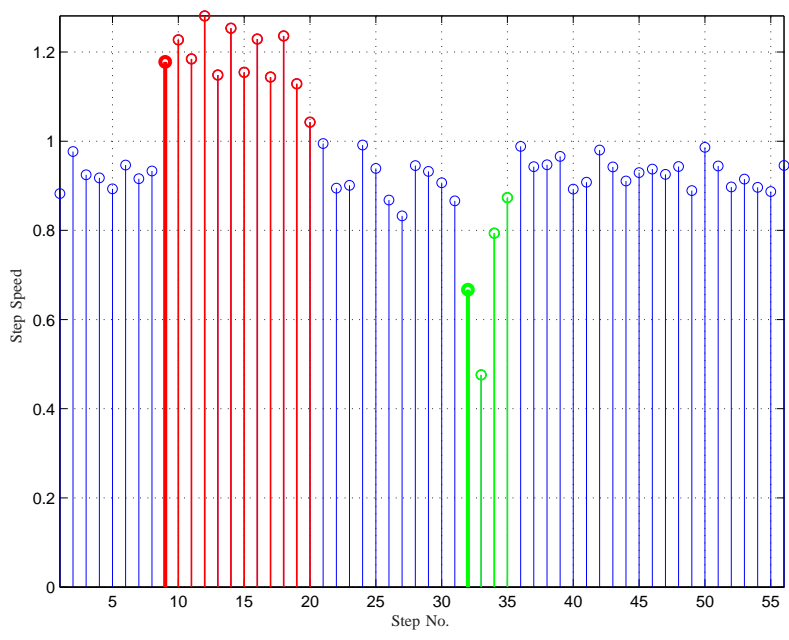

Fig. 16. Speed at each step for Exp. 2. An external disturbance in the form of a forward push is applied on step 9 (thick line), and the speed of the subsequent ten steps is faster than the nominal. An external disturbance in the form of a backward push is applied on step 32 (thick line), and the speed of the subsequent three steps is slower than the nominal. In both cases nominal speed is recovered. Interestingly, it takes more steps for the robot to lose energy and slow down rather than for the robot to gain energy and speed up. This indicates that very little energy is lost at impact.

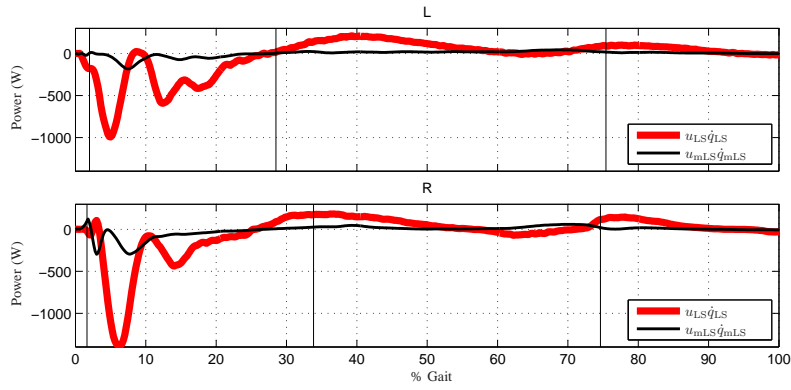

Fig. 17. Power plot of Exp. 3 implementing a fixed point obtained by optimizing for $c_{m t}$ (40). The plots are obtained by averaging the power over 77 steps (39 steps with the left leg as stance and 38 steps with the right as stance). The vertical lines indicate mean phase transition instants. Most of the negative work is done by the compliance instead of the actuator.

We thus stepped away momentarily from the problem of fast walking and concentrated on achieving a higher fidelity implementation of the virtual constraints. We decided to use the full I/O linearizing controller (42), with correction polynomials as in (45). Although zero dynamics based controllers are great in theory, all experimental implementations to date had been with simple PD controllers (Westervelt et al., 2004; Morris et al., 2006). Zero dynamics controllers are often criticized for being overly dependent on the model being accurate, and for being too complex to implement in real time.

Results: We report, for the first time, an experimental implementation ${ }^{4}$ of the full compliant hybrid zero dynamics controller to successfully achieve walking on

\footnotetext{
${ }^{4}$ To enable computing all terms of the zero dynamics based controller within a $1 \mathrm{~ms}$ sample time, an extremely efficient matrix library based on C++ expression templates (Veldhuizen, 1995; Alexandrescu, 2001) was used.
} 


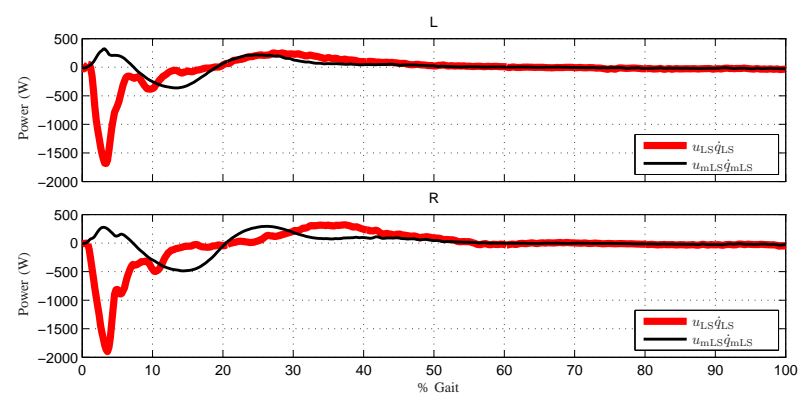

Fig. 18. Power plot for the hand-tuned virtual constraints experiment reported in (Grizzle et al., 2009). The plots are obtained by averaging the power over 32 steps. Immediately after impact and during the first $10 \%$ of the gait, we can see the motor actually fights the spring. In the rest of the gait, the motors do almost all the work.

MABEL. The virtual constraints of Exp. 1 are used here. The tracking accuracy obtained is far better than the feedforward plus PD controller used previously. The compliant zero dynamics controller, (32), (42), with the correction terms, (45), and with the modified event transition surface, (50), is deployed. The output coordinates are normalized to approximately the same magnitude for better conditioning of the decoupling matrix. Unlike in Section V-A, $u^{*}$ is computed from the dynamics directly and is not approximated. (Recall that in Section IV-A, $u^{*}$ was regressed against $\theta_{\mathrm{s}}$ to obtain a Bézier polynomial $u^{*}\left(\theta_{\mathrm{s}}, \beta_{\mathrm{s}}\right)$. This approximated $u^{*}$ along the periodic orbit as a function of $\theta_{\mathrm{S}}$ but provides no velocity correction.)

The tracking of the swing virtual constraints is at least as good as that obtained in Figure 11(a) with the PD controller and is not shown. Figures 12(a) and 12(b) compare the tracking of $q_{\text {Tor }}$ and $q_{\mathrm{mLS}_{\mathrm{st}}}$ under the effect of the PD and the compliant zero dynamics controllers, respectively. The tracking is greatly improved, where the error on $q_{\text {Tor }}$ reduced from a peak of $3.1^{\circ}$ to $2.4^{\circ}$, with a reduction in root mean square error (RMSE) from $1.44^{\circ}$ to $0.89^{\circ}$. Similarly, the error on $q_{\mathrm{mLS}}$ reduced from a peak of $59.1^{\circ}$ to $39.5^{\circ}$, with a reduction in RMSE from $29.82^{\circ}$ to $0.28^{\circ}$ (when scaled by a gear ratio of 31.42 , this translates to a reduction in error for $q_{\mathrm{LS}}$ from a peak of $1.9^{\circ}$ to $1.3^{\circ}$, with a reduction in RMSE from $0.95^{\circ}$ to $0.009^{\circ}$.)

Figure 19 illustrates the stance and swing $\mathrm{B}_{\text {spring }}$ values over a few steps. Notice that the $s c \rightarrow s i$ transition occurs more closely to the designed value. Figure 13(b) depicts the control torques at the actuators. These are noisier than with the PD control torques. This issue will be resolved in the next section.

The average walking speed for this experiment is $0.9 \mathrm{~m} / \mathrm{s}$. This is closer to the designed walking speed than in Exp. 1. The reduction in errors in the virtual constraint improves the correlation between the desired and realized walking speeds.

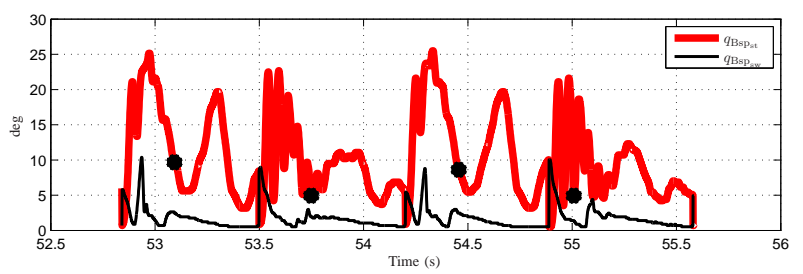

Fig. 19. B $_{\text {spring }}$ evolution for the compliant zero dynamics controller in Exp. 4. The asterisks indicate location of transition from the stance-compression $(s c)$ to the stance-decompression $(s d)$ phase. The transitions appear to be closer to the nominal value of $q_{\mathrm{Bsp}}=5^{\circ}$ when compared to the PD controller, Figure 15.

\section{E. Exp. 5: Fast Walking}

We return to the problem of achieving fast walking. A decentralized zero dynamics controller is implemented using the virtual constraints of Exp. 1. This is simply the zero dynamics controller as implemented in Exp. 4, with the off-diagonal elements of the decoupling matrix, $L_{g_{\mathrm{s}}} L_{f_{\mathrm{s}}} h$, set to zero. This was observed to reduce the 'cross-talk' in the control signal due to errors in one output being transferred to another. Figures 11(b) and 12(c) illustrate the virtual constraint tracking achieved with the decentralized zero dynamics controller. Figure 13 compares the torques obtained under the effect of the presented controllers. The torques for the decentralized zero dynamics controller are less noisy when compared to the torques for the compliant zero dynamics controller.

With this controller, MABEL started walking at around $1.15 \mathrm{~m} / \mathrm{s}$. The torso was gradually leaned forward to increase the speed. A top walking speed of $1.52 \mathrm{~m} / \mathrm{s}$ was achieved with a sustained walking speed of 1.5 $\mathrm{m} / \mathrm{s}$ (3.4 mph.) This made MABEL "the fastest walking biped of any size" in the world on October 31, 2009, and the record was held until April 22, 2010, when PETMAN reclaimed the speed record with a $1.97 \mathrm{~m} / \mathrm{s}$ walking gait; the video was posted to YouTube (Grizzle, 2010a).

Section VI-D compares the walking speed of several bipedal robots.

\section{DISCUSSION OF THE EXPERIMENTS}

This section discusses various aspects of the robot and the feedback controllers revealed by the experiments.

\section{A. Asymmetry}

The model used in the feedback designs has assumed a planar robot; in particular, this is predicated on the robot's dynamics being identical when the left leg is in stance or the right leg is in stance. The data shows clearly that this is false. The robot itself is nearly symmetric. The asymmetry arises from the boom used to constrain the robot to the sagittal plane. The facility housing the robot only permits a boom of length $2.25 \mathrm{~m}$ from the center of the floor to the center of the robot. The width of MABEL's hips is $0.24 \mathrm{~m}$, which is approximately $10 \%$ of the boom. For comparison, RABBIT has a boom of length $1.7 \mathrm{~m}$, with a hip width of $0.074 \mathrm{~m}$, which is 
approximately $4 \%$ of the boom. The robot plus boom may need to be modeled as a 3D system.

It was noted that the experimental walking speed for Exp. 1 in Section V-A was $1.0 \mathrm{~m} / \mathrm{s}$. The average speed with the left (inner) leg as stance is $0.98 \mathrm{~m} / \mathrm{s}$ while the average speed with the right (outer) leg as stance is 1.02 $\mathrm{m} / \mathrm{s}$. The ratio of left and right stance speeds is nearly equal to the ratio of distance of left and right legs to the center column.

\section{B. Impact Model}

For legged robots, the accuracy of the model of the leg end (foot) impact with the walking surface is difficult to ascertain and to improve. The vast majority of researchers adopt an instantaneous double support model, and use (Hurmuzlu and Marghitu, 1994) to build the corresponding mathematical model. Several researchers have used or proposed compliant ground models (Westervelt et al., 2007, p. 278), (Plestan et al., 2003; Canudas de Wit et al., 1995; Freeman and Orin, 1991; Bruneau and Ouezdou, 1997, 1999; Pascal, 1994; Wei et al., 1994, 1993), (Roussel, 1998, Chap. 5) yielding a double support phase of nonzero duration. Results exist in the literature in which rigid impact models have also been used to obtain non-trivial double support phases (Miossec and Aoustin, 2005; Roussel et al., 1998). While the compliant models seem more physically realistic, the uncertainty present in the parameters of such models does not necessarily yield a more accurate result, and it certainly does add considerable complexity to the model via numerical stiffness and / or non-Lipschitz continuous dynamics.

The bottom line is that when comparing theoretical predictions to experiments, the impact model should be considered as one possible source of error. In all of the experiments reported here, the robot walked faster than predicted by the dynamic model. The reference (Westervelt et al., 2004, Fig. 9) suggested that this could be accounted for by scaling the post-impact velocity predicted by (19). The scaling is performed here in such a way that the post-impact velocity still respects the constraints (16), (17), (18). The impact scaling is achieved by replacing $\dot{q}_{\mathrm{e}}^{+}$with $\eta \dot{q}_{\mathrm{e}}^{+}$in these constraints, where $\eta$ is an impact scaling factor. Table I shows various steady-state walking speeds for different values of the impact scaling factor. It is notable that for the simulation to match the experimental walking speed, we require $\eta=0.966$, a change of less than $4 \%$ to the impact map. Figure 20 compares the nominal walking experiment with a simulation with this impact scaling factor.

\section{Exp. 3: Efficient Walking}

As mentioned in Section V-C, the experimentally realized $c_{m t}$ is approximately three times the designed value.

One may suppose that this discrepancy is due to the existence of non-negligible motion of the motor shaft
TABLE I

EFFECT OF IMPACT MAP SCALING ON WALKING SPEED.

\begin{tabular}{cc}
\hline Impact map scaling factor $\eta$ & Steady-state walking speed \\
\hline 1.0 & $0.80 \mathrm{~m} / \mathrm{s}$ \\
0.99 & $0.86 \mathrm{~m} / \mathrm{s}$ \\
0.98 & $0.92 \mathrm{~m} / \mathrm{s}$ \\
0.97 & $0.98 \mathrm{~m} / \mathrm{s}$ \\
0.966 & $1.00 \mathrm{~m} / \mathrm{s}$ \\
0.96 & $1.04 \mathrm{~m} / \mathrm{s}$ \\
\hline
\end{tabular}

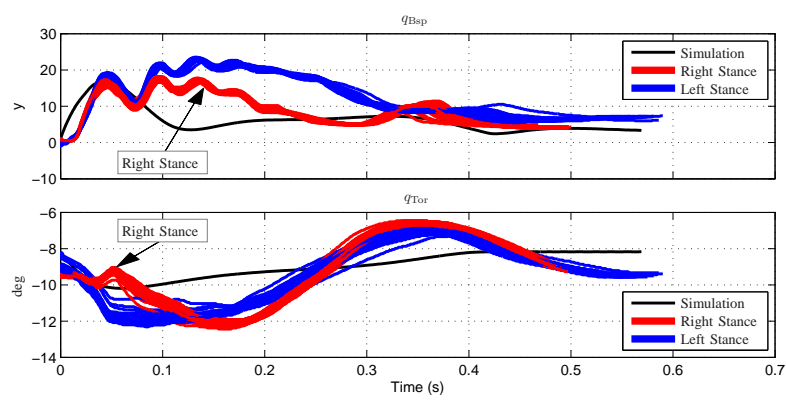

Fig. 20. This figure shows the plots of the $\mathrm{B}_{\text {spring }}$ and the Torso separately for the left and right legs as stance for Exp. 1 and compares with a simulation of the nominal fixed point with an impact scaling factor, $\eta=0.966$.

during the $s c$ and $s$ d phases, a consequence of the inability of the actuator to function as an ideal brake. However, this is not the case. Detailed examination of the mechanical work performed in the $s \mathrm{c}$ and $s \mathrm{~d}$ phases reveals that the stance motor leg-shape already does little work in the experiment. Introducing a brake in the experimental setup would then have little effect in improving the $c_{m t}$ value. After several simulations, perturbing different parts of the model, such as increasing friction and introducing cable stretch, we have observed that both friction and cable stretch contribute significantly to increase the specific cost of mechanical transport.

Table II illustrates the (mechanical) energy efficiency of several bipedal robots, and is sorted by $c_{m t}$. Although the experimentally obtained $c_{m t}$ value for MABEL is not as good as the designed value, it is 12 times better than that of Honda's ASIMO, over 2.75 times better than RABBIT and twice better than the hand-tuned virtual constraints based controller on MABEL. This puts MABEL's energy efficiency within a factor of two of T.U. Delft's Denise and a factor of three of the Cornell Biped, none of which can step over obstacles or run; it is also within a factor of two of the MIT Spring Flamingo which can easily step over obstacles but cannot run, and within a factor of three of humans, who can do all of the above..

\section{Exp. 5: Fast Walking}

In order to compare MABEL's walking performance with other bipedal robot designs and control methods, Table III lists robot parameters, peak walking speed, 
TABLE II

EFFICIENCY NUMBERS FOR VARIOUS BIPEDAL ROBOTS AND VARIOUS CONTROLLERS ON MABEL.

\begin{tabular}{lc}
\hline Robot & $\mathbf{c}_{\mathbf{m t}}$ \\
\hline Honda's ASIMO* & 1.60 \\
RABBIT (Westervelt, 2003, Sec. 6.5.1) & 0.38 \\
MABEL - Hand designed VC (Grizzle et al., 2009) & 0.29 \\
MABEL - 0.8 m/s FP, HZD Ctrl (Exp. 4) & 0.18 \\
MABEL - 0.8 m/s FP (Exp. 1) & 0.15 \\
MABEL - $c_{m t}=0.0385$ FP (Exp. 3) & $\mathbf{0 . 1 4}$ \\
T.U. Delft's Denise* (Collins and Ruina, 2005) & 0.08 \\
MIT's Spring Flamingo (Collins and Ruina, 2005) & 0.07 \\
Cornell Biped* (Collins et al., 2005) & 0.055 \\
McGeer's Dynamite (Collins and Ruina, 2005) & 0.04 \\
*3D, autonomous and untethered.
\end{tabular}

and the dimensionless velocity ${ }^{5}$. The table is sorted by peak speed. Of note is the bipedal robot RunBot (Manoonpong et al., 2007; Geng et al., 2006), which is the fastest walker measured by dimensionless velocity and leg lengths per second. MABEL was the fastest walker in terms of absolute speed from October 31, 2009 until April 22, 2010, when PETMAN took the record.

Notice that MABEL and it's predecessor, RABBIT, are the only ones in this list without ankles and feet. It has been suggested in (Lee and Piazza, 2009) that ankles and even toes, in humans, are very useful to provide a push-off to increase speed. The effect of pushoff in bipedal robots is studied in (Kuo, 2002) and is established as an energy efficient way to increase speed.

\section{E. Cable Stretch}

The differentials in MABEL's drivetrain, c.f. Figure 2, are realized by a series of cables and pulleys. The reader is referred to (Hurst, 2008; Park et al., 2010) for details. The robot was designed under the assumption that the cables undergo zero deformation, and this assumption has been used in developing the dynamic model of the robot that we used for control design. In the experiments, it has been observed that there is significant cable stretch. A representative plot of the cable stretch in the leg angle is shown in Figure 21, where the variable $q_{\mathrm{LA}}+q_{\mathrm{mLA}} / 23.53$ is plotted in degrees. If the cables were rigid, this variable would be identically zero ${ }^{6}$.

\section{F. Zeroing the Virtual Constraints}

The theorems supporting the method of virtual constraints are easier to prove when an input-output linearizing controller such as (42) is used (Morris and Grizzle, 2009; Westervelt et al., 2007; Poulakakis and Grizzle, 2009b). In practice, the benefits are achieved by any controller that realizes the constraints $h\left(q_{\mathrm{s}}\right)=H_{0}^{\mathrm{s}} q_{\mathrm{s}}-$ $h_{d}\left(\theta_{\mathrm{s}}\right)$ with "sufficient accuracy". This can be formalized using high-gain feedback via singular perturbations (Viola, 2008). On RABBIT the actuators were connected

\footnotetext{
${ }^{5}$ The dimensionless velocity serves as a speed metric and is defined as the square root of the Froude number, with the Froude number being the ratio of the centrifugal force due to motion about the foot and the weight of the robot (Vaughan and O'Malley, 2005).

${ }^{6}$ Encoders are present to directly measure both $q_{\mathrm{LA}}$ and $q_{\mathrm{mLA}}$.
}

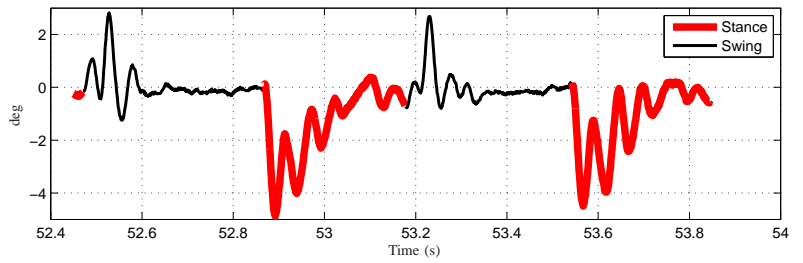

Fig. 21. Cable stretch as measured by $q_{\mathrm{LA}}-q_{\mathrm{mLA}} / 23.53$ for the right (outer) leg over two consecutive swing and stance phases for the fast walking experiment, Exp. 5. Negative cable stretch values are not to be interpreted as cable compression. Cable differentials require two sets of cables to 'pull' in either direction. The negative cable stretch values in the figure refer to cable stretch in the second cable.

to the robot's linkage through $50: 1$ gear ratios, and it turned out that a high-gain PD implementation resulted in sufficiently accurate realization of the virtual constraints. However, on MABEL, this simple controller was inadequate for fast gaits, motivating the implementation of an input-output linearizing controller. We believe that three things limited our ability to increase the PD gains in (43) sufficiently high to realize the constraints: lower gear ratios in the drivetrain ${ }^{7}$; encoder 'noise' when estimating derivatives; and the additional compliance arising from the cable stretch discussed above.

\section{CONCLUSION}

MABEL contains springs in its drivetrain for the purposes of enhancing energy efficiency and agility of dynamic locomotion. This paper has presented a novel analytical design method to realize the potential of the springs. An extensive set of experiments was performed to illustrate and confirm important aspects of the feedback design.

A HZD-based controller was designed to achieve asymptotically stable walking while recruiting the compliance in the robot's drivetrain to perform most of the negative work required to decelerate the downward motion of the robot's center of mass after impact, instead of the actuators. This not only improved the energy efficiency of walking, but also made the gait more natural looking. Stability analysis of the walking gait was performed using the method of Poincaré.

The analytically derived control law was experimentally validated on MABEL. The controller was demonstrated to be robust to external disturbances as well as to significant differences between the design model and the actual robot. In particular, the cables used to realize the differentials in the robot's drivertrain exhibited considerable stretch in the experiments, none of which was considered in the design model. Due to the observations made as part of these experiments, a more accurate model incorporating cable stretch has been presented in (Park et al., 2010).

${ }^{7}$ MABEL has a gear ratio of $23.53: 1$ and $31.42: 1$ for leg angle and leg-shape coordinates respectively. For comparision, RABBIT has a gear ratio of $50: 1$ at both the knees and hips. 
TABLE III

TOP WALKING SPEEDS OF BIPEDAL ROBOTS; THIS TABLE IS MOTIVATED BY (MANOONPONG ET AL., 2007, FigURE 1).

Mass (Kg)

Leg Length (m)

Peak Speed $(\mathrm{m} / \mathrm{s})$

Dimensionless Velocity

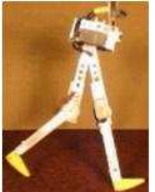

RunBot

0.53

0.23

0.80

0.53

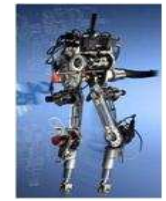

RABBIT

32.00

0.80

1.20

0.43

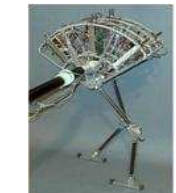

Spring Flamingo

14.20

0.90

1.25

0.42

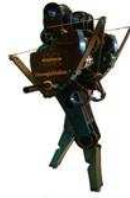

MABEL

65.00

1.00

1.50

0.48

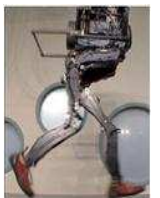

PETMAN

1.97

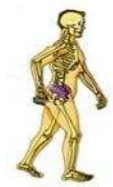

Olympic Record

$\approx 70.00$

$0.9 \approx 1.15$

4.60

$1.4 \approx 1.5$
A walking gait was designed to optimize for the energetic cost of mechanical transport $c_{m t}$ and then experimentally evaluated on MABEL. Even though MABEL has no feet, the experimentally realized $c_{m t}$ is 12 times better than that of ASIMO, approximately thrice as good as RABBIT, and twice as good as a hand-designed virtual-constraint-based controller that we had previously implemented on MABEL. This puts MABEL's energy efficiency within a factor of two of T.U. Delft's Denise, and a factor of three of the Cornell Biped, which are specifically designed mechanically for efficient walking. This demonstrates the interplay of mechanical design and control design in achieving higher efficiency.

For the first time, a real-time implementation of a complete hybrid zero dynamics based controller has been demonstrated in experiments. The tracking accuracy attained is far better than that of simple PD controllers used in prior experiments on RABBIT and MABEL. This removed the restriction of hybrid zero dynamics to theory or simulation, and establishes hybrid zero dynamics based controllers in the experimental domain.

A controller was implemented on MABEL that realized a sustained walking speed of $1.5 \mathrm{~m} / \mathrm{s}(3.4 \mathrm{mph})$. This made MABEL "the fastest robotic bipedal walker of any size" as of October 31, 2009, and the record was held until April 22, 2010.

This work has experimentally demonstrated a novel control design that preserves natural dynamics and has established MABEL as a successful stable, efficient and fast walker. This sets a very important preliminary stage for running on flat ground and for walking on uneven ground. Future research will be directed towards obtaining analytical and experimental results in these areas.

\section{Acknowledgments}

We wish to thank G. Buche for his many contributions to the design of the electronics, power supply and safety interlock systems. His prior experience with RABBIT was instrumental in us arriving at a much safer and more functional test facility. J. Koncsol is thanked for his selfless dedication to our project. His weekly visits to the laboratory and the generous sharing of his engineering experience contributed invaluably to the experiments reported here. B. Morris is acknowledged for his assistance in setting up the initial embedded computing environment, in addition to his contributions to the theoretical underpinnings of our work, as cited in the text. Last but not least, we are deeply indebted to J. Hurst for designing MABEL. We hope that this paper has confirmed many of his expectations for the robot.

\section{REFERENCES}

Alexander, R. (1990). Three uses for springs in legged locomotion. The International Journal of Robotics Research, 9(2):53-61.

Alexandrescu, A. (2001). Modern C++ Design: Generic Programming and Design Patterns Applied. AddisonWesley, Boston, MA.

Brown, B. and Zeglin, G. (1998). The bow leg hopping robot. In IEEE International Conference on Robotics and Automation.

Bruneau, O. and Ouezdou, F. B. (1997). Compliant contact of walking robot feet. In Proc. of third ECPD International Conference on Advanced Robotics, Intelligent Automation and Active Systems, Bremen, Germany.

Bruneau, O. and Ouezdou, F. B. (1999). Distributed ground/walking robot interaction. Robotica, 17(3):313-323.

Canudas de Wit, C., Olsson, H., Astrom, K., and Lischinsky, P. (1995). A new model for control of systems with friction. IEEE Transactions on Automatic Control, 40(3):419-425.

Chevallereau, C., Abba, G., Aoustin, Y., Plestan, F., Westervelt, E. R., de Wit, C. C., and Grizzle, J. W. (2003). Rabbit: a testbed for advanced control theory. IEEE Control Systems Magazine, 23(5):57-79.

Collins, S., Ruina, A., Tedrake, R., and Wisse, M. (2005). Efficient bipedal robots based on passivedynamic walkers. Science, 307:1082-1085.

Collins, S. H. and Ruina, A. (2005). A bipedal walking robot with efficient and human-like gait. In IEEE International Conference on Robotics and Automation, pages 1983-1988, Barcelona, Spain.

Freeman, P. S. and Orin, D. E. (1991). Efficient dynamic simulation of a quadruped using a decoupled treestructure approach. The International Journal of Robotics Research, 10(6):619-627.

Geng, T., Porr, B., and Wörgötter, F. (2006). Fast biped 
walking with a sensor-driven neuronal controller and real-time online learning. The International Journal of Robotics Research, 25(3):243-259.

Geppert, L. (2004). QRIO, the robot that could. In IEEE Spectrum, volume 41, pages 34-37.

Geyer, H., Seyfarth, A., and Blickhan, R. (2006). Compliant leg behaviour explains basic dynamics of walking and running. Proceedings of The Royal Society, 273:2861-2867.

Grizzle, J. W. (2010). Dynamic leg locomotion. Youtube Channel: http://www.youtube.com/ DynamicLegLocomotion.

Grizzle, J. W., Abba, G., and Plestan, F. (2001). Asymptotically stable walking for biped robots: Analysis via systems with impulse effects. IEEE Transactions on Automatic Control, 46(1):51-64.

Grizzle, J. W., Chevallereau, C., and Shih, C.-L. (2008). HZD-based control of a five-link underactuated $3 \mathrm{~d}$ bipedal robot. In IEEE Conference on Decision and Control, Cancun, Mexico.

Grizzle, J. W., Hurst, J., Morris, B., Park, H.-W., and Sreenath, K. (2009). MABEL, a new robotic bipedal walker and runner. In American Control Conference, pages 2030-2036, Saint Louis, MO, USA.

Hobbelen, D., de Boer, T., and Wisse, M. (2008). System overview of bipedal robots flame and tulip: tailormade for limit cycle walking. In IEEE/RSJ International Conference on Intelligent Robots and Systems, pages 2486-2491, Nice, France.

Hobbelen, D. G. E. and Wisse, M. (2008). Ankle actuation for limit cycle walkers. The International Journal of Robotics Research, 27(6):709-735.

Hodgins, J. K. and Raibert, M. H. (1990). Biped gymnastics. The International Journal of Robotics Research, 9(2):115-128.

Hurmuzlu, Y. and Marghitu, D. B. (1994). Rigid body collisions of planar kinematic chains with multiple contact points. The International Journal of Robotics Research, 13(1):82-92.

Hurst, J. W. (2008). The Role and Implementation of Compliance in Legged Locomotion. $\mathrm{PhD}$ thesis, Carnegie Mellon University.

Hurst, J. W., Chestnutt, J. E., and Rizzi, A. A. (2007). Design and philosophy of the bimasc, a highly dynamic biped. In IEEE International Conference on Robotics and Automation, pages 1863-1868, Roma, Italy.

Hurst, J. W. and Rizzi, A. A. (2008). Series compliance for an efficient running gait. IEEE Robotics \& Automation Magazine, 15(3):42-51.

Iida, F., Rummel, J., and Seyfarth, A. (2007). Bipedal walking and running with compliant legs. In IEEE International Conference on Robotics and Automation, pages 3970-3975, Roma, Italy.

Iida, F., Rummel, J., and Seyfarth, A. (2008). Bipedal walking and running with spring-like biarticular muscles. Journal of Biomechanics, 41(3):656-667.

Isidori, A. (1995). Nonlinear Control Systems. Springer-
Verlag, Berlin, third edition.

Kaneko, K., Kanehiro, F., Kajita, S., Yokoyama, K., Akachi, K., Kawasaki, T., Ota, S., and Isozumi, T. (2002). Design of prootype humanoid robotics platform for HRP. In Proceedings of the 2002 IEEE/RSJ International Conference on Intelligent Robots and Systems, pages 2431-2436, Lausanne, Switzerland.

Koechling, J. and Raibert, M. H. (1993). How Fast Can a Legged Robot Run?, volume Se of NATO ASI Series, chapter 3, pages 239-269. Springer-Verlag.

Kuo, A. D. (2002). Energetics of actively powered locomotion using the simplest walking model. Journal of Biomechanical Engineering, 124(1):113-120.

Lee, C. R. and Farley, C. T. (1998). Determinants of the center of mass trajectory in human walking and running. The Journal of Experimental Biology, 201(21):2935-2944.

Lee, S. S. M. and Piazza, S. J. (2009). Built for speed: musculoskeletal structure and sprinting ability. The Journal of Experimental Biology, 212(22):3700-3707.

Manchester, I. R., Mettin, U., Iida, F., and Tedrake, R. (2009). Stable dynamic walking over rough terrain: Theory and experiment. In International Symposium on Robotics Research.

Manoonpong, P., Geng, T., Kulvicius, T., Porr, B., and Wörgötter, F. (2007). Adaptive, fast walking in a biped robot under neuronal control and learning. Public Library of Science Computational Biology, 3(7):13051320.

Miossec, S. and Aoustin, Y. (2005). A simplified stability study for a biped walk with underactuated and overactuated phases. The International Journal of Robotics Research, 24(7):537-551.

Morris, B. and Grizzle, J. W. (2005). A restricted Poincaré map for determining exponentially stable periodic orbits in systems with impulse effects: Application to bipedal robots. In IEEE Conference on Decision and Control, pages 4199-206, Seville, Spain.

Morris, B. and Grizzle, J. W. (2006). Hybrid invariance in bipedal robots with series compliance. In IEEE Conference on Decision and Control, San Diego, California, USA.

Morris, B. J. and Grizzle, J. W. (2009). Hybrid invariant manifolds in systems with impulse effects with application to periodic locomotion in bipedal robots. IEEE Transactions on Automatic Control, 54(8):1751 $-1764$.

Morris, B. J., Westervelt, E. R., Chevallereau, C., Buche, G., and Grizzle, J. W. (2006). Achieving Bipedal Running with RABBIT: Six Steps Toward Infinity, volume 340 of Lecture Notes in Control and Information Sciences, pages 277-297. Springer Berlin / Heidelberg.

Park, H.-W., Sreenath, K., Hurst, J. W., and Grizzle, J. W. (2010). Identification and dynamic model of a bipedal robot with a cable-differential-based compliant drivetrain. Technical Report CGR 10-06, University of Michigan Control Group Report. 
Pascal, O. (1994). Contribution à la manipulation fine et étude de la phase d'impact. $\mathrm{PhD}$ thesis, Institut national polytechnique de Grenoble.

Plestan, F., Grizzle, J. W., Westervelt, E. R., and Abba, G. (2003). Stable walking of a 7-dof biped robot. IEEE Transactions on Robotics and Automation, 19(4):653-668.

Poulakakis, I. (2008). Stabilizing Monopedal Robot Running: Reduction-by-Feedback and Compliant Hybrid Zero Dynamics. PhD thesis, The University of Michigan.

Poulakakis, I. and Grizzle, J. W. (2009a). Modeling and control of the monopedal robot thumper. In IEEE International Conference on Robotics and Automation, pages 3327-3334, Kobe, Japan.

Poulakakis, I. and Grizzle, J. W. (2009b). The spring loaded inverted pendulum as the hybrid zero dynamics of an asymmetric hopper. IEEE Transactions on Automatic Control, 54(8):1779-1793.

Pratt, G. A. (2000). Legged robots at mit: what's new since raibert? IEEE Robotics \& Automation Magazine, 7(3):15-19.

Pratt, J., Chew, C.-M., Torres, A., Dilworth, P., and Pratt, G. (2001). Virtual model control: An intuitive approach for bipedal locomotion. The International Journal of Robotics Research, 20(2):129-143.

Pratt, J. E. and Pratt, G. A. (1998). Exploiting natural dynamics in the control of a planar bipedal walking robot. In Proceedings of the Thirty-Sixth Annual Allerton Conference on Communication, Control and Computing, Monticello, Illinois, USA.

Rebula, J. R., OConnor, S. M., and Kuo, A. D. (2009). Human walking and running: It's all in the redirection. In Dynamic Walking Conference.

Roussel, L. (1998). Génération de trajectoires de marche optimales pour un robot bipède. $\mathrm{PhD}$ thesis, Institut National Polytechnique de Grenoble.

Roussel, L., Canudas-De-Wit, C., and Goswami, A. (1998). Generation of energy optimal complete gait cycles for biped robots. In IEEE International Conference on Robotics and Automation, volume 3, pages 2036-2041, Leuven, Belguim.

Russ Tedrake, T. W. Z. and Seung, H. S. (2005). Learning to walk in 20 minutes. In In Proceedings of the Fourteenth Yale Workshop on Adaptive and Learning Systems, Yale University, New Haven, CT.

Sabourin, C., Bruneau, O., and Buche, G. (2006). Control strategy for the robust dynamic walk of a biped robot. The International Journal of Robotics Research, 25(9):843-860.

Sakagami, Y., Watanabe, R., Aoyama, C., Matsunaga, S., Higakiand, N., and Fujimura, K. (2002). The intelligent ASIMO: system overview and integration. In Proceedings of the IEEE International Conference on Intelligent Robots and Systems, volume 3, pages 2478-2483.

Schaub, T., Scheint, M., Sobotka, M., Seiberl, W., and Buss, M. (2009). Effects of compliant ankles on bipedal locomotion. In IEEE International Conference on Robotics and Automation, pages 2761-2766, Kobe, Japan.

Shiriaev, A. S., Freidovich, L. B., and Gusev, S. V. (2010). Transverse linearization for controlled mechanical systmes with several passive degrees of freedom. IEEE Transactions on Automatic Control, 55(4):893-906.

Shiriaev, A. S., Perram, J. W., and de Wit, C. C. (2005). Constructive tool for orbital stabilization of underactuated nonlinear systems: Virtual constraints approach. IEEE Transactions on Automatic Control, 50(8):1164-1176.

Song, G. and Žefran, M. (2006). Underactuated dynamic three-dimensional bipedal walking. In IEEE International Conference on Robotics and Automation, pages 854-859, Orlando, Florida, USA.

Takum, T., Hayashi, S., and Hosoda, K. (2008). 3d bipedal robot with tunable leg compliance mechanism for multi-modal locomotion. In IEEE/RSJ International Conference on Intelligent Robots and Systems, pages 1097-1102, Nice, France.

Vanderborght, B., Ham, R. V., Verrelst, B., Damme, M. V., and Lefeber, D. (2008a). Overiview of the lucy project: Dynamic stabilization of a biped powered by pneumatic artificial muscles. Advanced Robotics, 22(10):1027-1051.

Vanderborght, B., Verrelst, B., Ham, R. V., Damme, M. V., Beyl, P., and Lefeber, D. (2008b). Development of a compliance controller to reduce energy consumption for bipedal robots. Autonomous Robots, 24(4):419-434.

Vaughan, C. L. and O'Malley, M. J. (2005). Froude and the contribution of naval architecture to our understanding of bipedal locomotion. Gait \& Posture, 21(3):350 - 362.

Veldhuizen, T. (1995). Expression templates. In $C++$ Report, volume 7, pages 26-31.

Viola, G. (2008). Control of Underactuated Mechanical Systems via Passivity-Based and Geometric Techniques. PhD thesis, Università degli Studi di Roma "Tor Vergata".

Vukobratović, M. and Borovac, B. (2004). Zero-moment point-thirty five years of its life. International Journal of Humanoid Robotics, 1(1):157-73.

Wei, Q., Dayawansa, W., and Krishnaprasad, P. (1994). Approximation of dynamical effects due to impact on flexible bodies. In American Control Conference, pages 1841-1845, Baltimore, MD.

Wei, Q., Krishnaprasad, P., and Dayawansa, W. (1993). Modeling of impact on a flexible beam. In IEEE Conference on Decision and Control, volume 2, pages 1377-1382, San Antonio, TX.

Westervelt, E. R. (2003). Toward a Coherent Framework for the Control of Planar Biped Locomotion. $\mathrm{PhD}$ thesis, University of Michigan.

Westervelt, E. R., Buche, G., and Grizzle, J. W. (2004). Experimental validation of a framework for the design 
of controllers that induce stable walking in planar bipeds. The International Journal of Robotics Research, 23(6):559-582.

Westervelt, E. R., Grizzle, J. W., Chevallereau, C., Choi, J. H., and Morris, B. (2007). Feedback Control of Dynamic Bipedal Robot Locomotion. Taylor \& Francis/CRC Press, Boca Raton, FL.

Westervelt, E. R., Grizzle, J. W., and Koditschek, D. E. (2002). Zero dynamics of underactuated planar biped walkers. In International Federation of Automatic Control, Barcelona, Spain.

Westervelt, E. R., Grizzle, J. W., and Koditschek, D. E. (2003). Hybrid zero dynamics of planar biped walkers. IEEE Transactions on Automatic Control, 48(1):4256.

Yang, T., Westervelt, E. R., Schmiedeler, J. P., and Bockbrander, R. A. (2008). Deisgn and control of a planar bipedal robot ernie with parallel knee compliance. Autonomous Robots, 25(4):317-330.

\section{APPENDIX A}

\section{BÉZIER POLYNOMIALS FOR SUbPHASES}

This section develops a framework for virtual constraints with subphases and provides details on how the Bézier polynomials for the stance subphases are obtained.

\section{A. Framework for Virtual Constraints with Subphases}

Let $\mathcal{P}$ be an index set representing the subphases of the virtual constraints, and let $p \in \mathcal{P}$ denote a particular subphase. Similarly, let $\mathcal{V}$ be an index set representing the virtual constraints, and let $v \in \mathcal{V}$ denote a particular virtual constraint. In each subphase, the virtual constraint will be an $M^{t h}$ order Bézier polynomial parametrized by $\theta_{\mathrm{s}}$, where, $\theta_{v}^{p+}, \theta_{v}^{p-}$ are the starting and ending values of $\theta_{\mathrm{S}}$ respectively within phase $p$ of virtual constraint $v$. It is convenient to normalize each $\theta_{\mathrm{s}}$ to $[0,1]$ by defining

$$
s_{v}^{p}:=\frac{\theta_{\mathrm{s}}-\theta_{v}^{p+}}{\theta_{v}^{p-}-\theta_{v}^{p+}},
$$

where $\theta_{\mathrm{s}}$ goes from $\theta_{v}^{p+}$ to $\theta_{v}^{p-}$ during phase $p \in \mathcal{P}$. Then, if $\alpha_{v}^{p}$ is the vector of Bézier coefficients, then the desired evolution of the virtual constraint $v$ for phase $p$ can be expressed as

$$
h_{v}^{d, p}\left(\theta_{\mathrm{s}}\right)=\sum_{k=0}^{M} \alpha_{v}^{p}(k) \frac{M !}{k !(M-k) !} s_{v}^{p k}\left(1-s_{v}^{p}\right)^{M-k} .
$$

To ensure $\mathcal{C}^{k}$ continuity between successive subphases $p_{1}, p_{2} \in \mathcal{P}$, a standard property of Bézier curves specifies how the last $k+1$ parameters of $\alpha_{v}^{p_{1}}$ and the first $\mathrm{k}+1$ parameters of $\alpha_{v}^{p_{2}}$ must be related (Westervelt et al., 2007, p. 139). Next, it can be convenient to treat successive subphases $p_{1}, p_{2}$, defined over domains $\left[\theta_{v}^{p_{1}+}, \theta_{v}^{p_{1}-}\right],\left[\theta_{v}^{p_{2}+}, \theta_{v}^{p_{2}-}\right]$ respectively, as a single combined phase $p_{1} p_{2}$ with domain $\left[\theta_{v}^{p_{1}+}, \theta_{v}^{p_{2}-}\right]$. This is particularly useful for virtual constraints that do not require the resolution of multiple subphases and enables parameterizing with a single Bézier polynomial over both subphases. To do this, we impose $\theta_{v}^{p_{1}-}=\theta_{v}^{p_{2}-}$, $\theta_{v}^{p_{2}+}=\theta_{v}^{p_{1}+}$, and $\alpha_{v}^{p_{1}}=\alpha_{v}^{p_{2}}=: \alpha_{v}^{p_{1} p_{2}}$ in our general framework for virtual constraints with subphases. Thus we obtain $\left.s_{v}^{p_{1} p_{2}}\right|_{p_{1}}=s_{v}^{p_{1}}$ and $\left.s_{v}^{p_{1} p_{2}}\right|_{p_{2}}=s_{v}^{p_{2}}$, with $s_{v}^{p_{1} p_{2}}$ monotonically increasing from zero to one over both phases $p_{1}, p_{2}$. With this setup, the transition event from subphase $p_{1}$ to $p_{2}$ no longer has any effect on the combined phase $p_{1} p_{2}$. This phase combination can be extended to more than two phases, and in fact all of the phases of a controlled variable can be combined, resulting in a single virtual constraint over the entire stance phase with no subphases.

\section{B. Stance Motor Leg-shape Virtual Constraint}

In the motor-compresssion phase, the motor legshape position is given by a Bézier polynomial parametrized by $s_{\mathrm{mLS}}^{m c}$, with coefficients $\alpha_{\mathrm{mLS}}^{m c} \mathrm{~s}_{\mathrm{st}}$ and $\theta_{\mathrm{mLS}}^{m c+}=\theta^{\mathrm{s}+}, \theta_{\mathrm{mLS}}^{m c-}=\theta^{m c-}$. The boundary conditions $\left(q_{\mathrm{mLS}_{\mathrm{st}}}^{m c+}=q_{\mathrm{mLS}}^{\mathrm{s}+}, \dot{q}_{\mathrm{mLS}}^{m c+}=\dot{q}_{\mathrm{sLS}}^{\mathrm{s}+}\right)$, and $\left(q_{\mathrm{mLS}}^{m c-}=q_{\mathrm{mLS}}^{s c}, \dot{q}_{\mathrm{mLS}}^{m c-}=0\right)$ specify the starting and the ending two coefficients, and the middle coefficients are free to be chosen as part of the control design , and are chosen to smoothly transition between $q_{\mathrm{mLS}}^{\mathrm{s}+}$ and $q_{\mathrm{mLS}}^{s c}$.

In the stance-compression phase, the motor leg-shape position is given by a Bézier polynomial parametrized by $s_{\mathrm{mLS}}^{s c}$, with coefficients $\alpha_{\mathrm{mLS}}^{s c}$ all equal to $q_{\mathrm{mLS}}^{s c}$, and $\theta_{\mathrm{mLS}_{\mathrm{st}}}^{s c+}=\theta^{\mathrm{s}+}, \theta_{\mathrm{mLS}}^{s c-}=\theta^{s c-}$.

In the stance-injection phase, the motor legshape position is given by a Bézier polynomial parametrized by, $s_{\mathrm{mLS}}^{s i}$, with coefficients $\alpha_{\mathrm{mLS}}^{s i}$ and $\theta_{\mathrm{mLS}}^{\text {si }}=\theta^{\text {st }}, \theta_{\mathrm{mLS}}^{s i-}=\theta_{\mathrm{st}}=$ The boundary conditions $\left(q_{\mathrm{mLS}_{\mathrm{st}}}^{s i+}=q_{\mathrm{mLS}}^{s c}, \dot{q}_{\mathrm{mLS}}^{s i+}=0\right)$, $\left(q_{\mathrm{mLS}}^{s i-}=q_{\mathrm{mLS}}^{s c-}, \dot{q}_{\mathrm{sLS}}^{s i-}=0\right)$ specify the starting and ending two parameters of $\alpha_{\mathrm{mLS}}^{s i}$ with the rest being free parameters to be chosen as part of control design.

In the stance-decompression phase, the motor legshape position is given by a Bézier polynomial parametrized by $s_{\mathrm{mLS}_{\mathrm{st}}}^{s d}$, with coefficients $\alpha_{\mathrm{mLS}}^{s d}$ all equal to $q_{\mathrm{mLS}}^{s c-}$, and $\theta_{\mathrm{mLS}}^{s d+}=\theta^{s d+}, \theta_{\mathrm{mLS}_{\mathrm{st}}}^{s d-}=\theta^{\mathrm{s}-}$.

Thus, parameters of only $\alpha_{\mathrm{mLS}}^{s i}$ are available to be chosen as part of control design. For notation purposes, we define $\alpha_{\mathrm{mLS}_{\mathrm{st}}}:=\alpha_{\mathrm{mLS}}^{\text {si }}$.

\section{Torso Virtual Constraint}

The motor-compression, stance-compression, and stance-injection phases, are combined into a single phase by setting $\theta_{\text {Tor }}^{m c+}=\theta_{\text {Tor }}^{s c+}=\theta_{\text {Tor }}^{s i+}=\theta^{\mathrm{s}+}, \theta_{\text {Tor }}^{m c-}=\theta_{\text {Tor }}^{s c-}=$ $\theta_{\text {Tor }}^{s i-}=\theta^{s i-}$, and $\alpha_{\text {Tor }}^{m c}=\alpha_{\text {Tor }}^{s c}=\alpha_{\text {Tor }}^{s i}=: \alpha_{\text {Tor }}$. Thus the torso evolution in this combined phase is given by a Bézier polynomial parametrized by $s_{\text {Tor }}$, with coefficients $\alpha_{\text {Tor }}$.

The desired torso evolution in the stancedecompression phase is given by a Bézier polynomial 
parametrized by $s_{\text {Tor }}^{s d}$, with coefficients $\alpha_{\text {Tor }}^{s d}$ all equal to $q_{\text {Tor }}^{\text {s- }}$, and $\theta_{\text {Tor }}^{s d+}=\theta^{s d+}, \theta_{\text {Tor }}^{s d-}=\theta^{\text {s- }}$.

\section{Swing Leg Virtual Constraints}

For the swing virtual constraints, all subphases are combined into one by setting $\theta_{\mathrm{LA}_{\mathrm{sw}}}^{m c+}=\theta_{\mathrm{LA}_{\mathrm{sw}}}^{s c+}=\theta_{\mathrm{LA}_{\mathrm{sw}}}^{s i+}=$ $\theta_{\mathrm{LA}_{\mathrm{sw}}}^{s d+}=\theta^{\mathrm{s}+}, \theta_{\mathrm{LA}_{\mathrm{sw}}}^{m c-}=\theta_{\mathrm{LA}_{\mathrm{sw}}}^{s c-}=\theta_{\mathrm{LA}}^{s i-}=\theta_{\mathrm{LA}_{\mathrm{sw}}}^{s d-}=\theta^{\mathrm{s}-}$, and $\alpha_{\mathrm{LA}}^{m c}=\alpha_{\mathrm{LA}}^{s c} A_{\mathrm{sw}}=\alpha_{\mathrm{LA}}^{s i}=\alpha_{\mathrm{Lw}}^{s d}=: \alpha_{\mathrm{LA}_{\mathrm{sw}}}$. Thus the evolution of the swing leg angle is given by a Bézier polynomial parametrized by $s_{\mathrm{LA}_{\mathrm{sw}}}$, with coefficients $\alpha_{\mathrm{LA}_{\mathrm{sw}}}$. In a completely similar manner, the swing motor leg shape is parameterized by $\alpha_{\mathrm{mLS}_{\mathrm{sw}}}$.

The Bézier coefficients that are not specified above are free parameters in the virtual constraints, and are specified by control design. These parameters can be put in a vector as,

$$
\alpha_{\mathrm{s}}=\left[\begin{array}{c}
\alpha_{\mathrm{mLS}_{\mathrm{st}}} \\
\alpha_{\mathrm{LA}_{\mathrm{sw}}} \\
\alpha_{\mathrm{mLS}_{\mathrm{sw}}} \\
\alpha_{\mathrm{Tor}}
\end{array}\right] .
$$

\section{APPENDIX B}

\section{OPTIMIZATION DETAILS}

Equality and inequality constraints are used during the optimization process to ensure that the closedloop system yields a desired behavior. These constraints could be limits on peak actuator torques, joint space constraints, unilateral ground contact forces, speed of walking, ground clearance, etc. Further, the general form of the virtual constraints chosen in Section III-A is assumed to be satisfied on the periodic orbit. This enables integrating the stance zero dynamics over the reduced-order closed-loop system dynamics (established in Section III-C) thereby reducing the computation time significantly. The details of the optimization algorithm are described below.

\section{Algorithm}

1) Select $\theta_{\mathrm{s}}^{-*}, q_{\mathrm{Bsp}_{\mathrm{st}}}^{-*}, q_{\mathrm{LA}_{\mathrm{sw}}}^{-*}, q_{\mathrm{mLS}}^{-*}{ }_{\mathrm{sw}}, q_{\mathrm{Tor}}^{-*}$. Determine $q_{L A_{\mathrm{st}}}^{-*}$ using (25). Determine $q_{m L S_{\mathrm{st}}}^{-*}$ by a NewtonRhapson search to satisfy $p_{\text {toe }}^{v-*}=0$ as this ensures that the impact condition, $q^{-*} \in S_{\mathrm{s} \rightarrow \mathrm{s}}$, is met.

2) Select $\dot{\theta}_{\mathrm{s}}^{-*}, \quad \dot{q}_{\mathrm{Bsp}_{\mathrm{st}}}^{-*}, \quad \dot{q}_{\mathrm{LA}}^{-*}, \quad \dot{q}_{\mathrm{mLS}}^{-*}, \quad$ Choose $\dot{q}_{\mathrm{mLS}}^{-*}=0, \dot{q}_{\text {Tor }}^{-*}=0$ to satisfy the virtual constraints described in section III-A. Determine $\dot{q}_{L A_{\mathrm{st}}}^{-*}$ using (25).

3) Using the stance-to-stance transition function, $\Delta_{\mathrm{s} \rightarrow \mathrm{s}}$, obtain $x_{\mathrm{s}}^{+*}=\left(q_{\mathrm{s}}^{+*} ; \dot{q}_{\mathrm{s}}^{+*}\right)$, the state corresponding to the beginning of the subsequent stance phase.

4) Calculate $\theta_{\mathrm{s}}^{+*}, \dot{\theta}_{\mathrm{s}}^{+*}$ using (25). Set $\theta_{m c}^{-*}=$ $\theta_{\mathrm{s}}^{+*}+0.05\left(\theta_{\mathrm{s}}^{-*}-\theta_{\mathrm{s}}^{+*}\right)$, and $\theta_{s i}^{-*}=\theta_{\mathrm{s}}^{+*}+$ $0.7\left(\theta_{\mathrm{s}}^{-*}-\theta_{\mathrm{s}}^{+*}\right)$ corresponding to $5 \%$ and $70 \%$ of the stance phase respectively, and set $q_{\mathrm{mLS}}^{s c *}=$ $q_{\mathrm{mLS}}^{\mathrm{s}+*}+\operatorname{sgn}\left(\dot{q}_{\mathrm{mLS}}^{\mathrm{s}+*} \mathrm{~s}_{\mathrm{st}}\right) \max \left(20^{\circ},\left|\dot{q}_{\mathrm{mLS}}^{\mathrm{s}+*}\right|\right)$. This facilitates the use of the first $5 \%$ of the gait to drive
TABLE IV

THE LIST OF INDEPENDENT PARAMETERS TO BE DETERMINED BY OPTIMIZATION. THE CHOICE OF THESE PARAMETERS IS NON-UNIQUE, AND DEPENDS ON THE ALGORITHM AND CONSTRAINTS EMPLOYED IN OPTIMIZATION.

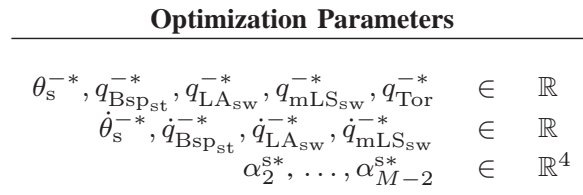

non-zero post-impact motor leg shape velocity to zero, and the last $30 \%$ of the gait to hold the torso constant in preparation for impact.

5) Select $\alpha_{\mathrm{s}, 2}^{*}, \ldots, \alpha_{\mathrm{s}, M-2}^{*}$. Calculate $\alpha_{\mathrm{s}, 0}^{*}, \alpha_{\mathrm{s}, 1}^{*}$ to satisfy the post-impact conditions, and calculate $\alpha_{\mathrm{s}, M-1}^{*}, \alpha_{\mathrm{s}, M}^{*}$ to satisfy the pre-impact conditions. Set $\alpha_{\mathrm{mLS}_{\mathrm{st}}, 2}^{m c *}, \ldots, \alpha_{\mathrm{mLS}}^{m c *}, M-2$ to get a smooth transition between $q_{\mathrm{mLS}}^{\mathrm{s}+*}$ and $q_{\mathrm{mLS}}^{s c *}$.

6) Integrate the stance dynamics for the motorcorrection phase, $\Sigma_{m c}$, and the stance-compression phase, $\Sigma_{s c}$, until the spring undergoes maximum compression and reaches a decompressed value of five degrees. Set this value of $\theta_{\mathrm{s}}$ as $\theta_{\mathrm{s}}^{s c-}$. Integrate the stance dynamics through the stance-injection, $\Sigma_{s i}$, and stance-decompression, $\Sigma_{s d}$, phases to obtain $x_{\mathrm{s}}^{-}$.

7) Evaluate the cost function $J$, equality constraints $E Q$, inequality constraints INEQ.

8) Iterate the above steps until $J$ is minimized and the equality and inequality constraints are satisfied.

Equality constraints, EQ

- Error associated with finding a fixed point $\| x_{\mathrm{s}}^{-}-$ $x_{\mathrm{s}}^{-*} \|=0$.

- Toe position of the swing leg at the end of the step $p_{\text {toe }_{\mathrm{sw}}}^{v}=0$.

\section{Inequality constraints, INEQ}

- Magnitude of the minimum normal force at the stance leg to be positive, $\min \left(F_{\mathrm{st}}^{N}\right)>0$.

- Maximum of magnitude of coefficient of friction less than one, $\left|\max \left(F_{\mathrm{st}}^{T} / F_{\mathrm{st}}^{N}\right)\right|<0.6$.

- Walking speed greater than $0.7 \mathrm{~m} / \mathrm{s}$.

- Swing leg toe profile to be above the ground throughout the stance phase.

- Swing leg angle not to exceed $220^{\circ}$.

- Stance leg angle not less than $140^{\circ}$.

- Range of travel of torso less than $5^{\circ}$. 